\title{
Distribution of Energy Levels of Quantum Free Particle on the Liouville Surface and Trace Formulae
}

\author{
Pavel M. Bleher ${ }^{1}$, Denis V. Kosygin ${ }^{2}$, Yakov G. Sinai ${ }^{2}$ \\ 1 Department of Mathematical Sciences, Indiana University - Purdue University at Indianapolis, \\ 402 N. Blackford Street, Indianapolis, IN 46202, USA \\ 2 Princeton University, Department of Mathematics, Princeton, NJ 08544, USA
}

Received: 6 June 1994/in revised form: 2 November 1994

Abstract: We consider the Weyl asymptotic formula

$$
\#\left\{E_{n} \leqq R^{2}\right\}=\frac{\text { Area } Q}{4 \pi} R^{2}+n(R),
$$

for eigenvalues of the Laplace-Beltrami operator on a two-dimensional torus $Q$ with a Liouville metric which is in a sense the most general case of an integrable metric. We prove that if the surface $Q$ is non-degenerate then the remainder term $n(R)$ has the form $n(R)=R^{1 / 2} \theta(R)$, where $\theta(R)$ is an almost periodic function of the Besicovitch class $B^{1}$, and the Fourier amplitudes and the Fourier frequencies of $\theta(R)$ can be expressed via lengths of closed geodesics on $Q$ and other simple geometric characteristics of these geodesics. We prove then that if the surface $Q$ is generic then the limit distribution of $\theta(R)$ has a density $p(t)$, which is an entire function of $t$ possessing an asymptotics on a real line, $\log p(t) \sim-C_{ \pm} t^{4}$ as $t \rightarrow \pm \infty$. An explicit expression for the Fourier transform of $p(t)$ via Fourier amplitudes of $\theta(R)$ is also given. We obtain the analogue of the Guillemin-Duistermaat trace formula for the Liouville surfaces and discuss its accuracy.

\section{Introduction}

The question about the relation of a quantum system to its classical limit has been discussed since the moment of appearance of quantum mechanics. Recently this question became popular again both in physics and mathematics due to the theory of quantum chaos. It turns out that statistical properties of quantum energy levels depend strongly on the ergodic properties of the underlying classical system. As an important and instructive example one may think of the Laplace-Beltrami operator on a Riemannian manifold and of the geodesic flow in the manifold as its classical counterpart. This case has been widely discussed in many physical and mathematical papers (see, e.g., $[1-11,13-17,20,21]$ ).

Let $X$ be a smooth compact Riemannian manifold and let $-\Delta$ be its LaplaceBeltrami operator. The eigen-states of a free quantum particle moving on $X$ are the eigenfunctions of the Laplace-Beltrami operator, i.e., the solutions of the equation 


$$
-\Delta f=E f \text {. }
$$

The positive square root of this operator, $\sqrt{-\Delta}$ is a selfadjoint elliptic pseudodifferential operator of order one. Its spectrum is discrete and each eigenvalue has finite multiplicity. If we enumerate them in non-decreasing order then we have

$$
0=R_{0}<R_{1} \leqq R_{2} \leqq \cdots \leqq R_{k} \leqq \cdots \rightarrow \infty \text { as } k \rightarrow \infty
$$

Note that the corresponding eigenvalue $E_{k}$ of the Laplace-Beltrami operator is equal to $R_{k}^{2}$. One of the possible approaches to the study of statistics of the spectrum is to consider the trace of the operator $\psi(-\Delta)$ for various functions $\psi$ (see $[1,2,10$, 11]). Properties of this trace, for some particular function $\psi$, are closely related to the properties of the Green function of the wave equation

$$
u_{t}=i \sqrt{-\Delta} u
$$

on $X$ which allows to use this relation reciprocally (see $[9,10,13,14]$ ).

The behavior of the sum $\sum_{k=1}^{\infty} \psi\left(R_{k}\right)$ depends on the geometry of $X$ and in particular on the structure of the set of closed geodesics on $X$. Before formulating corresponding results let us introduce some definitions. Denote by $M$ the unit cotangent bundle over $X$ and denote by $\xi$ the Hamilton vector field on $M$ whose generating function is the symbol of $\sqrt{-\Delta}$. We recall that the Hamilton flow generated by $\xi$ is the geodesic flow acting on $M$ whose trajectories, when projected onto $X$, are geodesics. Let $\gamma$ be a periodic trajectory of period $T$. The map $\exp (T \xi)$ maps $M$ onto $M$ and it is the identity map on $\gamma$. Thus for $x \in \gamma$, the differential $d(\exp T \xi)_{x}$ is a linear map on $T_{x}$ onto inself such that the tangent space to $\gamma$ is its one-dimensional eigenspace. By $P_{\gamma}$ we denote the induced linear transformation of the orthogonal complement to the tangent space to $\gamma$ at the point $x \in \gamma . P_{\gamma}$ is the linearization of the Poincaré map defined on a small submanifold of codimension 1 transversal to $\gamma$. Two linear Poincaré maps taken at two distinct points $x$ and $x^{\prime}$ of $\gamma$ are conjugate. A periodic trajectory $\gamma$ is called nondegenerate if it is isolated and $I-P_{\gamma}$ is invertible. A periodic trajectory $\{\gamma(t), 0 \leqq t \leqq T\}, \gamma(T)$, is called primitive if it does not contain periodic trajectories of smaller period, i.e., $\gamma(0) \neq \gamma\left(T^{\prime}\right)$ for $0<T^{\prime}<T$. Each periodic trajectory is obviously an iterate of a primitive periodic trajectory. Given a periodic trajectory we denote by $T_{\gamma}$ its period and by $T_{\gamma}^{*}$ the period of the primitive trajectory of which it is the iterate.

If all periodic trajectories of $\xi$ are nondegenerate then as shown in [9], the difference of the distributions

$$
\left(1+\sum_{\lambda^{2} \in \operatorname{spec}(-\Delta)} \exp (i \lambda t)\right)-\left(\sum_{\text {all periodic trajectories }} i^{i^{\text {ind }} \gamma} T_{\gamma}^{*}\left|I-P_{\gamma}\right|^{-1 / 2} \delta\left(t-T_{\gamma}\right)\right)
$$

is a locally $L^{1}$-summable function of $t$ on the interval $(0, \infty)$. Here $\operatorname{ind}_{\gamma}$ is the Maslov index of the trajectory $\gamma$. (Note that $\operatorname{ind}_{\gamma}$ is an integer mod 4 ).

When $X$ is a surface of constant negative curvature the exact expression of this function is given by the Selberg trace formula $([16,18])$.

Surfaces with completely integrable geodesic flow are in a sense opposite to the last mentioned case. Namely, the phase space $M$ is foliated onto $n$-dimensional invariant tori with quasi-periodic motion defined by $\xi$. When the ratio of frequencies of this motion on a torus is rational then this torus is filled up with periodic trajectories of the same period. It is not surprising that the exact expressions of the type 
of Selberg trace formula are not true any longer. We remark that general methods described in $[9,10,13,14]$ allows one to obtain some trace-type formulae even in the case when closed trajectories fill submanifolds of nontrivial codimension. In this paper we take a different approach.

In the present paper we continue to study spectral properties of the LaplaceBeltrami operator on Liouville surfaces started in [15] and [6]. The geodesic flow on a Liouville surface is completely integrable and it is believed that this is the most general class of surfaces for which this is the case (cf. [22]). It is to be noted also that contrary to surfaces of revolution a generic Liouville surface has no symmetries.

Let $\left(Q, d q^{2}\right)$ be a two-dimensional compact closed Riemannian manifold homeomorphic to a torus. We may think of $Q$ as being represented by unit square with coordinates $q_{1}, q_{2}, 0 \leqq q_{1}, q_{2} \leqq 1$. A surface $Q$ is called Liouville if its metric $d q^{2}$ has the form

$$
d q^{2}=\left[U_{1}\left(q_{1}\right)-U_{2}\left(q_{2}\right)\right]\left(d q_{1}^{2}+d q_{2}^{2}\right),
$$

where $U_{1}\left(q_{1}\right), U_{2}\left(q_{2}\right)$ are periodic functions of period 1 satisfying the inequality

$$
U_{1}\left(q_{1}\right)-U_{2}\left(q_{2}\right)>0 \text { for all } q=\left(q_{1}, q_{2}\right), 0 \leqq q_{1}, q_{2} \leqq 1 .
$$

The Laplace-Beltrami operator $-\Delta$ defined by this metric is given by the formula

$$
-\Delta f=-\left[U_{1}\left(q_{1}\right)-U_{2}\left(q_{2}\right)\right]^{-1}\left(\frac{\partial^{2} f}{\partial q_{1}^{2}}+\frac{\partial^{2} f}{\partial q_{2}^{2}}\right) .
$$

This is a non-negative selfadjoint operator in the Hilbert space $L^{2}(Q, \mu(d q))$ having a discrete spectrum. Here

$$
\mu(d q)=\left(U_{1}\left(q_{1}\right)-U_{2}\left(q_{2}\right)\right) d q_{1} d q_{2} .
$$

The first eigenvalue of $-\Delta$ is simple and corresponds to the constant eigenfunction. If we enumerate the eigenvalues in a non-decreasing order then we have

$$
0=E_{0}<E_{1} \leqq E_{2} \leqq \cdots \leqq E_{k} \leqq \cdots \rightarrow \infty \text { as } k \rightarrow \infty
$$

Denote by $N(x)$ the number of eigenvalues $E_{k}$ which are not greater than $x$,

$$
N(x)=\#\left\{k: E_{k} \leqq x\right\} .
$$

Then due to the Weyl asymptotic formula (see [14])

$$
N(x)=\frac{\operatorname{Area} Q}{4 \pi} x+n(x)
$$

where $\frac{n(x)}{x} \rightarrow 0$ as $x \rightarrow \infty$. The behavior of the remainder term $n(x)$ as $x \rightarrow \infty$ for Liouville surfaces was studied in [15], and it was shown that under a certain condition of non-degeneracy on the Liouville surface, the function $\theta(R)=R^{-1 / 2} n\left(R^{2}\right)$ is an almost periodic function of the Besicovitch class $B^{1}$. In addition, if the surface $\left(Q, d q^{2}\right)$ is generic then the probability distribution corresponding to the function $\theta(R)$ possesses nice analytical properties. In particular, it has a density which is an entire function of its argument decaying on the real axis at least as $\exp \left(-|t|^{(16 / 9)-\varepsilon}\right)$ when $t \rightarrow \pm \infty$. 
Surfaces of revolution are Liouville surfaces but they do not satisfy the nondegeneracy conditions assumed in [15]. This case was considered separately in [3-7] (see especially [6]). It was proven that under similar conditions of non-degeneracy the function $\theta(R)$ is an almost periodic function of the Besicovitch class $B^{2}$, and the set of the Fourier frequencies of $\theta(R)$ coincides with the geodesic spectrum of $Q$, i.e., with the set of the lengths of all closed geodesics on $Q$. The Fourier amplitudes of $\theta(R)$ have a nice geometric interpretation as well. In addition, for generic surfaces of revolution, which are characterized by linear independence of the lengths of all primitive geodesics on $Q$, the density of the distribution corresponding to $\theta(R)$ is an entire function which decays on the real axis roughly as $\exp \left(-\lambda t^{4}\right)$ when $|t| \rightarrow \infty$.

It is to be noted that almost periodic functions appear naturally in various problems of number theory (see e.g. [4, 7, 12, 15] and references therein). In particular, one interesting application of the theory of almost periodic functions to the problem of the distribution of primes in arithmetic progressions was described in [19]. As was shown in [15], the spectrum of the Laplace-Beltrami operator on a Liouville surface has a nice geometric interpretation in terms of points of two-dimensional lattices. Then the study of the function $N(x)$ is reduced to the counting lattice points in a planar domain which is essentially a problem in number theory.

In this paper we extend the results of [6] to the case treated in [15] and we obtain a refined estimate on the rate of decay of the distribution density corresponding to the function $\theta(R)$. This proves a conjecture stated in [15]. In addition, we prove a trace formula for the Liouville surfaces. The structure of this paper is the following. All necessary properties of the geodesic flow on a Liouville surface are described in Sect. 2 and the main results are formulated in Sect. 3. Section 4 contains a reduction of the main results to corresponding problems in number theory. The main steps of the proofs are exposed in Sect. 5 with some technical lemmas proven in Sect. 6. In Sect. 7 we prove some properties of the kernel of fractional integration of order $3 / 2$ which were used in the other parts of the paper.

\section{Properties of the Geodesic Flow of Liouville Surface}

Consider a torus $Q$ with the Liouville metric (1.1). Denote by $Q^{r}, 0 \leqq r \leqq \infty$, the space of pairs of $C^{r}$-functions $\left(U_{1}, U_{2}\right)$ on a circle satisfying (1.2) and the following condition:

A1. For each function $U_{1}, U_{2}$ there are exactly two points where its derivative vanishes. One of these points is its maximum, and the other one is minimum. Both of these points are non-degenerate (i.e., the second derivative does not vanish at these points).

$Q^{r}$ is a topological space with the $C^{r}$-topology. One may think of points in the space $Q^{r}$ as of corresponding Liouville surfaces, defined by means of the functions $U_{1}, U_{2}$ and the formula (1.1). The structure of the geodesic flow on a surface $Q$ with the metric from the space $Q^{r}$ was described in detail in [15] and for proofs we refer the reader to that paper.

The geodesic flow is embedded into the Hamilton flow acting on the cotangent bundle $T^{*} Q$ and defined by the Hamiltonian

$$
H(p, q)=\frac{1}{2\left(U_{1}\left(q_{1}\right)-U_{2}\left(q_{2}\right)\right)}\left(p_{1}^{2}+p_{2}^{2}\right)
$$


where

$$
p_{i}=\left(U_{1}\left(q_{1}\right)-U_{2}\left(q_{2}\right)\right) d q_{i}, i=1,2 .
$$

This Hamilton flow possesses the first integral different from the energy integral,

$$
S(p, q)=\frac{1}{2\left(U_{1}\left(q_{1}\right)-U_{2}\left(q_{2}\right)\right)}\left(U_{2}\left(q_{2}\right) p_{1}^{2}+U_{1}\left(q_{1}\right) p_{2}^{2}\right),
$$

and thus it is completely integrable. The function $S(p, q)$ (as well as $H$ ) is a homogeneous function of order 2 of the momentum $p=\left(p_{1}, p_{2}\right)$. We associate with $S(p, q)$ the quantum operator

$$
\sigma=\left[U_{1}\left(q_{1}\right)-U_{2}\left(q_{2}\right)\right]^{-1}\left(U_{2}\left(q_{2}\right) \frac{\partial^{2}}{\partial q_{1}^{2}}+U_{1}\left(q_{1}\right) \frac{\partial^{2}}{\partial q_{2}^{2}}\right) .
$$

A direct computation shows that $\Delta$ and $\sigma$ commute.

Introduce the constants $c_{1}, c_{2}, c_{3}, c_{4}, m_{1}, M_{1}, m_{2}, M_{2}$ as

$$
\begin{array}{ll}
c_{1}=\max _{0 \leqq x \leqq 1} U_{1}(x)=U_{1}\left(M_{1}\right), & c_{2}=\min _{0 \leqq x \leqq 1} U_{1}(x)=U_{1}\left(m_{1}\right), \\
c_{3}=\max _{0 \leqq x \leqq 1} U_{2}(x)=U_{1}\left(M_{2}\right), & c_{4}=\min _{0 \leqq x \leqq 1} U_{2}(x)=U_{1}\left(m_{2}\right),
\end{array}
$$

For $E>0$ and $c \in\left[c_{4}, c_{1}\right]$, define

$$
M_{E, c}=\left\{(p, q) \in T^{*} Q: H(p, q)=E, S(p, q)=c E\right\} .
$$

Due to the complete integrability, every set $M_{E, c}$ consists of two two-dimensional tori when $c \in\left(c_{4}, c_{3}\right) \cup\left(c_{2}, c_{1}\right)$, and of four two-dimensional tori when $c_{3}<c<$ $c_{2}$. The phase trajectories of the Hamiltonian system are windings over these tori, and the projection $\pi:(p, q) \rightarrow q$ maps them onto geodesics on the configuration torus $Q$.

In the case $c \in\left(c_{4}, c_{3}\right) \cup\left(c_{2}, c_{1}\right), \pi$ projects each component of $M_{E, c}$ onto either a horizontal or vertical strip, and the projection of any trajectory on $M_{E, c}$ is a geodesic which rotates along the strip and oscillates between its two boundaries. Two components of $M_{E, c}$ correspond to two possible directions of the rotation along the strip. If $c \in\left(c_{3}, c_{2}\right), \pi$ projects each component of $M_{E, c}$ in a one-to-one way onto the whole configurational torus $Q$, and every trajectory on $M_{E, c}$ is projected onto a geodesic, which is a curvilinear winding over $Q$. Four components of $M_{E, c}$ correspond in this case to four possible combinations of the direction of rotation along $q_{1}$ and $q_{2}$.

The values $c=c_{1}, c_{2}, c_{3}, c_{4}$ are exceptional: for $c=c_{1}, c_{4}$ the tori degenerate into two circles while for $c=c_{2}, c_{3}$ they degenerate into four cylinders corresponding to the direct product of the separatrix and the circle, with different directions of motion along separatrix and the circle (for more detailed description see [15]). In what follows we will call freely the components of $M_{E, c}$ when $c=c_{1}, c_{2}, c_{3}, c_{4}$ invariant tori as well.

The Hamiltonian flow can be described with the help of action-angle variables $I_{1}, I_{2}, \varphi_{1}, \varphi_{2}$. Let $\zeta_{1}, \zeta_{2}$ be the circles on $Q$ defined by the equations $q_{1}=M_{1}, q_{2}=$ $m_{2}$, respectively, with the orientation induced by the coordinate axes. For each invariant torus corresponding to a nonexceptional value of the parameter $c$ a connected component of its intersection with $\pi^{-1}\left(\zeta_{i}\right)$ is a cycle $\alpha_{i}$ homeomorphic to circle $(i=1,2)$. The canonical form $p d q=p_{1} d q_{1}+p_{2} d q_{2}$ determines uniquely an 
orientation of the cycle $\alpha_{i}$. Namely, the tangent vector to $\alpha_{i}$ is positively oriented if the value of $p d q$ on it is positive. The action variables $I_{1}, I_{2}$ related to the cycles $\alpha_{1}, \alpha_{2}$ are given by the formulae:

$$
I_{i}= \begin{cases}-\int_{\alpha_{i}} p d q, & \text { if } \pi: \alpha_{i} \rightarrow \zeta_{i} \text { is bijective and } \pi\left(\alpha_{i}\right) \text { and } \zeta_{i} \text { have different } \\ & \text { orientations } ; \\ \int_{\alpha_{i}} p d q, & \text { otherwise } .\end{cases}
$$

By $\varphi_{1}, \varphi_{2}$ we denote the angle variables canonically conjugate to $I_{1}, I_{2}$.

As follows from (2.1) and (2.2), $(p, q) \in M_{E, c}$ implies

$$
p_{1}^{2}=2 E\left(U_{1}\left(q_{1}\right)-c\right), \quad p_{2}^{2}=2 E\left(c-U_{2}\left(q_{2}\right)\right),
$$

hence the action variables are

$$
I_{1}(E, c)=\oint_{\pi\left(\alpha_{1}\right)} p_{1} d q_{1}=\left\{\begin{array}{l}
2(2 E)^{1 / 2} \underset{q_{1}: U_{1}\left(q_{1}\right)-c \geqq 0}{\int}\left(U_{1}\left(q_{1}\right)-c\right)^{1 / 2} d q_{1} \\
\text { when } c_{2}<c \leqq c_{1} \\
\pm(2 E)^{1 / 2} \int_{0}^{1}\left(U_{1}\left(q_{1}\right)-c\right)^{1 / 2} d q_{1} \\
\text { when } c_{4} \leqq c<c_{2}
\end{array}\right.
$$

and

$$
I_{2}(E, c)=\oint_{\pi\left(\alpha_{2}\right)} p_{2} d q_{2}=\left\{\begin{array}{l} 
\pm(2 E)^{1 / 2} \int_{0}^{1}\left(c-U_{2}\left(q_{2}\right)\right)^{1 / 2} d q_{2} \\
\text { when } c_{3}<c \leqq c_{1} ; \\
2(2 E)^{1 / 2} \int_{q_{2}: c-U_{2}\left(q_{2}\right) \geqq 0}\left(c-U_{2}\left(q_{2}\right)\right)^{1 / 2} d q_{2} \\
\text { when } c_{4} \leqq c<c_{3}
\end{array}\right.
$$

According to the definition, in formulae (2.4) and (2.5) we put the plus sign if the motion goes in the positive direction (with respect to a given coordinate) and the minus sign otherwise. More precisely, the formulae (2.4), (2.5) enable us to define $I_{1}, I_{2}$ as functions on the phase space $T^{*} Q$ which are constant on each invariant torus, and the value of which on the given torus is defined by (2.4), (2.5). It is noteworthy that the mapping which maps an invariant torus to the corresponding pair $\left(I_{1}, I_{2}\right)$ is one-to-one. Note also, that the actions $I_{1}(E, c), I_{2}(E, c)$ are multivalued functions of $E$ and $c$, namely, the values of $I_{1}$ and $I_{2}$ on the invariant tori corresponding to the same pair $(E, c)$ are equal in absolute value but may differ in sign.

The frequencies of the motion along an invariant torus defined by a pair $\left(I_{1}, I_{2}\right)$ are given by the formulae

$$
\omega_{1}(E, c)=\frac{\partial H}{\partial I_{1}}, \quad \omega_{2}(E, c)=\frac{\partial H}{\partial I_{2}}
$$

The frequencies $\omega_{1}(E, c), \omega_{2}(E, c)$ like the actions $I_{1}, I_{2}$ are multivalued functions of $E$ and $c$. Namely, frequencies of motion along invariant tori corresponding to the same pair $(E, c)$ are equal in absolute value but may differ in sign. We define the 
functions $\omega_{1}, \omega_{2}$ at $c=c_{1}, c_{2}, c_{3}, c_{4}$ by continuity. The functions $I_{i}(E, c), \omega_{i}(E, c)$ are homogeneous functions of $E$ of order $1 / 2$, hence

$$
I_{i}(E, c)=(2 E)^{1 / 2} f_{i}(c), \quad \omega_{i}(E, c)=(2 E)^{1 / 2} \xi_{i}(c),
$$

where $f_{i}(c)=I_{i}(1 / 2, c), \xi_{i}(c)=\omega_{i}(1 / 2, c)$. Recall that an invariant torus is called non-degenerate if on this torus,

$$
\operatorname{det}\left(\frac{\partial^{2} H}{\partial I_{k} \partial I_{l}}\right)=\operatorname{det}\left(\frac{\partial \omega_{k}}{\partial I_{l}}\right) \neq 0 .
$$

If the invariant torus is non-degenarate then in its small neighborhood the frequencies $\omega_{j}$ can serve as local coordinates, instead of the action variables. A straightforward computation gives

$$
\begin{aligned}
& \xi_{1}=\frac{f_{2}^{\prime}}{\Delta_{1}}, \quad \xi_{2}=-\frac{f_{1}^{\prime}}{\Delta_{1}} \\
& \xi_{1}^{\prime}=-f_{2} \frac{\Delta_{2}}{\Delta_{1}^{2}}, \quad \xi_{2}^{\prime}=f_{1} \frac{\Delta_{2}}{\Delta_{1}^{2}} ;
\end{aligned}
$$

where

$$
\Delta_{1}=f_{1} f_{2}^{\prime}-f_{2} f_{1}^{\prime}, \quad \Delta_{2}=f_{1}^{\prime} f_{2}^{\prime \prime}-f_{2}^{\prime} f_{1}^{\prime \prime}
$$

Hence

$$
\begin{aligned}
& \xi_{1} f_{1}^{\prime}+\xi_{2} f_{2}^{\prime}=0, \\
& \xi_{1} f_{1}+\xi_{2} f_{2}=1,
\end{aligned}
$$

and

$$
\xi_{1} \xi_{2}^{\prime}-\xi_{2} \xi_{1}^{\prime}=\frac{\Delta_{2}}{\Delta_{1}^{2}}
$$

This implies

$$
\operatorname{det}\left(\frac{\partial^{2} H}{\partial I_{k} \partial I_{l}}\right)(E, c)=\frac{\xi_{1} \xi_{2}^{\prime}-\xi_{2} \xi_{1}^{\prime}}{\Delta_{1}}=\frac{\Delta_{2}}{\Delta_{1}^{3}} .
$$

Observe that $\operatorname{det}\left(\frac{\partial^{2} H}{\partial I_{k} \partial I_{l}}\right)(E, c)$ does not depend on $E$. In the sequel we shall need the following functions:

$$
\begin{aligned}
& b(c)=\frac{f_{1}^{\prime} f_{2}^{\prime \prime}-f_{2}^{\prime} f_{1}^{\prime \prime}}{\left(f_{1}^{\prime 2}+f_{2}^{\prime 2}\right)^{3 / 2}} \\
& a(c)=\left(\xi_{1}^{2}+\xi_{2}^{2}\right)^{3 / 2} b(c)=\operatorname{det}\left(\frac{\partial^{2} H}{\partial I_{k} \partial I_{l}}\right)(E, c)
\end{aligned}
$$

and

$$
\begin{aligned}
& F_{1}(c)=\left\{\begin{array}{rr}
\frac{1}{2} f_{1}(c) & \text { when } c_{2} \leqq c \leqq c_{1} \\
f_{1}(c) & \text { when } c_{4} \leqq c \leqq c_{2}
\end{array}\right. \\
& F_{2}(c)=\left\{\begin{array}{cc}
f_{2}(c) & \text { when } c_{3} \leqq c \leqq c_{1} \\
\frac{1}{2} f_{2}(c) & \text { when } c_{4} \leqq c \leqq c_{3}
\end{array}\right.
\end{aligned}
$$


The functions $a(c), b(c)$ like $f_{1}, f_{2}, \xi_{1}, \xi_{2}$ are multivated functions of $c$ and their values on different branches at the same point $c$ are equal in absolute value but may differ in sign.

Denote by $W$ the set of all invariant tori with $E=1 / 2$. As was said above, every $\omega \in W$ is characterized by the value of the parameter $c_{4} \leqq c \leqq c_{1}$ and by the choice of one or two signs, which give the direction of rotation on $\omega$ along the axes $q_{1}, q_{2}$. It is to be noted that the tori $\omega \in W$ fill up the unit cotangent bundle over $Q$. Denote by $W_{+}$the set of $\omega \in W$ with positive direction of rotation.

Let $\mathscr{G}$ be the set of all nonzero oriented closed (in general, multiple) geodesics on $Q$. For $g \in \mathscr{G}$ denote by $\omega(g) \in W$ the invariant torus on which $\pi^{-1} g$ lies and by $c(g)$ the value of the parameter $c$ on $\omega(g)$. Let $n_{1}(g), n_{2}(g) \in \mathbb{Z}$ be the rotation numbers of $\pi^{-1} g$ along the axes $q_{1}, q_{2}$, respectively. If $\pi$ projects $\omega(g)$ onto the whole $Q$ (which occurs when $\left.c_{3} \leqq c(g) \leqq c_{2}\right)$ then we assign to $n_{1}(g), n_{2}(g)$ the signs of the direction of rotation of $g$ along the axes $q_{1}, q_{2}$, respectively. If $\pi$ projects $\omega(g)$ onto a strip along $q_{1}$ (which occurs when $c_{4} \leqq c(g)<c_{3}$ ) then we assign to $n_{1}(g)$ the sign of the direction of rotation of $g$ along $q_{1}$ and the sign + to $n_{2}(g)$. Observe that in this case $n_{2}(g)$ describes the number of oscillations of $g$ along the axis $q_{2}$ so it is natural that $n_{2}(g) \geqq 0$. Similarly, if $\pi$ projects $\omega(g)$ onto a band along $q_{2}$ (which occurs when $c_{2}<c(g) \leqq c_{1}$ ) we assign to $n_{2}(g)$ the sign of the direction of rotation of $g$ along $q_{2}$ and the sign + to $n_{1}(g)$. We denote by $\xi_{1}(g), \xi_{2}(g), f_{1}(g), f_{2}(g)$, respectively, the frequencies and the actions of the motion along $\omega(g)$.

If $g \in \mathscr{G}$ then the Hamiltonian flow is periodic on the invariant torus $\omega(g)$ and

$$
\xi_{1} / \xi_{2}=n_{1}(g) / n_{2}(g) \in \mathbb{Q} \cup\{ \pm \infty\} .
$$

We will call two closed geodesics equivalent if they correspond to the same torus $\omega(g)$, and we will denote by $G$ the set if geodesics $g \in \mathscr{G}$ factorized by this relation of equivalence. Remark that all equivalent closed geodesics have the same values of $n_{1}(g), n_{2}(g)$ and of the length

$$
|g|=\frac{n_{1}}{\xi_{1}}=\frac{n_{2}}{\xi_{2}}=n_{1} f_{1}+n_{2} f_{2},
$$

so $n_{1}(g), n_{2}(g)$ and $|g|$ can be viewed as functions on $G$.

\section{Formulation of the Main Results}

We begin with some definitions. We shall call a pair of real numbers $\alpha_{1}, \alpha_{2}$ diophantine if there exist $\tau>1$ and $C>0$ such that for any nonzero pair of integers $k_{1}, k_{2}$,

$$
\left|k_{1} \alpha_{1}+k_{2} \alpha_{2}\right| \geqq \frac{C}{|k|(\log |k|)^{\tau}}, \quad|k|=\left(k_{1}^{2}+k_{2}^{2}\right)^{1 / 2} .
$$

A number $\alpha$ is called diophantine if the pair $1, \alpha$ is diophantine. The complement to the set of diophantine pairs of numbers (as well as to the set of diophantine numbers) has zero Lebesgue measure. Real numbers $1=\lambda_{0}, \lambda_{1}, \lambda_{2}, \ldots$ are called linearly independent over $\mathbb{Z}$ if

$$
k_{0}+k_{1} \lambda_{1}+\cdots+k_{n} \lambda_{n}=0
$$

with $k_{i} \in \mathbb{Z}$ implies $k_{0}=\cdots=k_{n}=0$. 
A function $f(t)$ on the positive half-axis $\{t>0\}$ is called an almost periodic function of the Besicovitch class $B^{p}$ if for any positive $\varepsilon$ there exists a trigonometric polynomial

$$
P_{\varepsilon}(t)=\sum_{n=1}^{N_{\varepsilon}} a_{n, \varepsilon} \exp \left(i \lambda_{n, \varepsilon} t\right)
$$

such that

$$
\limsup _{T \rightarrow \infty} T^{-1} \int_{0}^{T}\left|f(t)-P_{\varepsilon}(t)\right|^{p} d t \leqq \varepsilon .
$$

In addition, a trigonometric series

$$
\sum_{n=1}^{\infty} a_{n} \exp \left(i \lambda_{n} t\right)
$$

is called the Fourier series of $f(t)$ with respect to $B^{p}$ if

$$
\lim _{N \rightarrow \infty} \limsup _{T \rightarrow \infty} T^{-1} \int_{0}^{T}\left|f(t)-\sum_{n=1}^{N} a_{n} \exp \left(i \lambda_{n} t\right)\right|^{p} d t=0 .
$$

The Fourier series of an almost periodic function is always well-defined and unique (see [17]).

Theorem 3.1. Let a Liouville surface $Q \in \mathscr{2}^{r}, r \geqq 5$, satisfy the following conditions:

(i) The function

$$
a(c)=\operatorname{det} d^{2} H(E, c), \quad d^{2} H=\left(\frac{\partial^{2} H}{\partial I_{k} \partial I_{l}}\right),
$$

has only finitely many zeroes and all these zeroes are zeroes of the first order.

(ii) If $c$ is a zero of $a(c)$ then the pairs $\left(\xi_{1}(c), \xi_{2}(c)\right)$ and $\left(f_{1}(c), f_{2}(c)\right)$ are diophantine.

(iii) The pairs $\left(f_{1}\left(c_{2}\right), f_{2}\left(c_{2}\right)\right)$ and $\left(f_{1}\left(c_{3}\right), f_{2}\left(c_{3}\right)\right)$ are diophantine,

(iv) The pairs $\left(\omega_{1}\left(c_{1}\right), \omega_{2}\left(c_{1}\right)\right)$ and $\left(\omega_{2}\left(c_{4}\right), \omega_{2}\left(c_{4}\right)\right)$ are diophantine.

Then the function $N(x)=\#\left\{k: E_{k} \leqq x\right\}$ has the representation

$$
N(x)=\frac{\text { Area } Q}{4 \pi} x+x^{1 / 4} \theta\left(x^{1 / 2}\right),
$$

where $\theta(R)$ is an almost-periodic function of the Besicovitch class $B^{1}$. The Fourier series of $\theta(R)$ with respect to $B^{1}$ is

$$
\theta(R)=\left(2 \pi^{3}\right)^{-1 / 2} \sum_{g \in G}|g|^{-3 / 2} x(g)^{-1 / 2} \sin \left(|g| R-\frac{\pi}{2} \text { ind } g-\frac{\pi}{4} \sigma(g)\right) .
$$

Here ind $g$ means the Maslov index of any geodesic from the class $g \in G$ and

$$
\varkappa(g)=\left|\operatorname{det} d^{2} H\right|, \quad \sigma(g)=\operatorname{sign} \operatorname{det} d^{2} H,
$$

where the value of $\operatorname{det} d^{2} H$ is taken on the torus $\omega(g)$.

It is noteworthy that the fulfillment of assumptions in Theorem 3.1 does not depend on the choice of the branches of the functions $a, f_{1}, f_{2}, \omega_{1}, \omega_{2}$. The almostperiodic function $\theta(R)$ was also considered by Berry and Tabor in [2]. As concerns 
the properties of the function $\theta(R)$, it is plausible that this function belongs actually to the Besicovitch class $B^{2}$ (and not only to $B^{1}$ as stated), and Theorem 4.3 below strongly supports this conjecture. Also the $B^{2}$ property of $\theta(R)$ is established for a class of surfaces of revolution, see [6]. Still in Theorem 3.1 we have a problem with proving the $B^{2}$ almost periodicity, when we estimate the difference between the function $\theta(R)$ and the function $\theta_{0}(R)$ of Theorem 4.3. Namely, to estimate this difference we use semiclassical quantization of the energy levels, and the usual Bohr-Sommerfeld quantization formula turns out to be insufficient for our purposes, because it does not work near unstable periodic orbits. So we derive a more sophisticated semiclassical quantization formula which works near unstable periodic orbits, and a difficult problem is to obtain a good uniform estimate of the error term in this formula, see [15] and [6a].

Denote by $G_{0}$ the subset of $G$ consisting of all closed geodesics (up to the equivalence) with non-negative relatively prime rotation numbers $n_{1}(g), n_{2}(g)$. In other words, $G_{0}$ is the set of all primitive closed geodesics on $Q$, up to the equivalence and to the choice of orientation.

Theorem 3.2. Let $Q$ be a Liouville surface which satisfies the assumptions of Theorem 3.1. Assume in addition that the numbers $\left\{|g|, g \in G_{0}\right\}$ are linearly independent over $\mathbb{Z}$. Then the limit distribution of the function $\theta(R)$ is absolutely continuous with respect to the Lebesque measure, and the density $p(t)$ of this distribution is an entire function of $t$ which has the following asymptotics on the real axis:

$$
\lim _{t \rightarrow \pm \infty}\left(-t^{-4} \log p(t)\right)=C_{ \pm}>0
$$

where $C_{ \pm}$are two (in general different) constants which can be expressed explicitly via geometrical characteristics of the geodesic flow (for an exact formula see (4.25) below).

Before formulating the exact statement concerning the trace formula on the Liouville surface we give a heuristic derivation of it. Let $\varphi(t)$ be a test function from the Schwartz space, i.e., $\varphi(t)$ decays faster than polynomially as $|t| \rightarrow \infty$, together with all its derivatives. Put

$$
F(x)=\sum_{R_{k} \in \operatorname{spec} \sqrt{-\Delta}} \varphi\left(x-R_{k}\right), \quad M(R)=N\left(R^{2}\right), \quad m(R)=n\left(R^{2}\right) .
$$

Then integrating by parts we have

$$
\begin{aligned}
F(x) & =\sum_{R_{k} \in \operatorname{spec} \sqrt{-\Delta}} \varphi\left(x-R_{k}\right)=\int_{-0}^{\infty} \varphi(x-y) d M(y)=\int_{-0}^{\infty} \varphi^{\prime}(x-y) M(y) d y \\
& =\frac{\operatorname{Area} Q}{4 \pi} \int_{0}^{\infty} \varphi^{\prime}(x-y) y^{2} d y+\int_{-0}^{\infty} \varphi^{\prime}(x-y) m(y) d y \\
& =\frac{\operatorname{Area} Q}{2 \pi} \int_{0}^{\infty} \varphi(x-y) y d y+\int_{-0}^{\infty} \varphi^{\prime}(x-y) y^{1 / 2} \theta(y) d y .
\end{aligned}
$$


Replacing $\theta(y)$ by its Fourier series (3.3) and neglecting the error term coming from this replacement (since the Fourier series of a function from $B^{1}$ represents the function only asymptotically at infinity) we obtain

$$
\begin{aligned}
F(x)= & \frac{\text { Area } Q}{2 \pi} \int_{0}^{\infty} \varphi(x-y) y d y+\left(2 \pi^{3}\right)^{-1 / 2} \sum_{g \in G}|g|^{-3 / 2} \varkappa(g)^{-1 / 2} \\
& \times \int_{-0}^{\infty} \varphi^{\prime}(x-y) y^{1 / 2} \sin \left(|g| y-\frac{\pi}{2} \text { ind } g-\frac{\pi}{4} \sigma(g)\right) d y .
\end{aligned}
$$

Now,

$$
\begin{aligned}
& \int_{-0}^{\infty} \varphi^{\prime}(x-y) y^{1 / 2} \sin \left(|g| y-\frac{\pi}{2} \text { ind } g-\frac{\pi}{4} \sigma(g)\right) d y \\
& =|g| \int_{-0}^{\infty} \varphi(x-y) y^{1 / 2} \cos \left(|g| y-\frac{\pi}{2} \text { ind } g-\frac{\pi}{4} \sigma(g)\right) d y+O\left(x^{-1 / 2}\right), \quad x \rightarrow \infty,
\end{aligned}
$$

so neglecting the error term $O\left(x^{-1 / 2}\right)$ in the last formula we arrive at

$$
\begin{aligned}
F(x)= & \frac{\operatorname{Area} Q}{2 \pi} \int_{0}^{\infty} \varphi(x-y) y d y+\left(2 \pi^{3}\right)^{-1 / 2} \sum_{g \in G}|g|^{-1 / 2} \chi(g)^{-1 / 2} \\
& \times \int_{-0}^{\infty} \varphi(x-y) y^{1 / 2} \cos \left(|g| y-\frac{\pi}{2} \operatorname{ind} g-\frac{\pi}{4} \sigma(g)\right) d y
\end{aligned}
$$

In the language of the theory of distributions this is equivalent to

$$
\begin{aligned}
\sum_{R_{k} \in \operatorname{spec} \sqrt{-\Delta}} \delta\left(x-R_{k}\right)= & \frac{\text { Area } Q}{2 \pi} x_{+}+x_{+}^{1 / 2}\left(2 \pi^{3}\right)^{-1 / 2} \sum_{g \in G}|g|^{-1 / 2} \varkappa(g)^{-1 / 2} \\
& \times \cos \left(|g| y-\frac{\pi}{2} \text { ind } g-\frac{\pi}{4} \sigma(g)\right),
\end{aligned}
$$

where

$$
x_{+}^{\lambda}= \begin{cases}x^{\lambda} & \text { for } x>0 \\ 0 & \text { for } x \leqq 0\end{cases}
$$

Passing to the Fourier transform we obtain that

$$
\begin{aligned}
\sum_{R_{k} \in \operatorname{spec} \sqrt{-\Delta}} e^{i R_{k} \xi}= & -\frac{\text { Area } Q}{2 \pi}(\xi+i 0)^{-2}+\left(2 \pi^{3}\right)^{-1 / 2} \sum_{g \in G \pm} \sum_{ \pm}|g|^{-1 / 2} \chi(g)^{-1 / 2} \\
& \times e^{ \pm \frac{\pi i}{4}(2 \text { ind } g+\sigma(g))} v(\xi \mp|g|),
\end{aligned}
$$

where $v(\xi)=\frac{\sqrt{\pi}}{2} e^{\frac{3 \pi i}{4}}(\xi+i 0)^{-3 / 2}$ is the Fourier transform of $x_{+}^{1 / 2}$. The formula (3.8) is certainly only approximate since in its derivation we neglected different error terms. The sense of the formula (3.8) is that it describes the principal singularities of the tempered distribution

$$
\chi(\xi)=\operatorname{tr} e^{i \xi \sqrt{-\Delta}}=\sum_{R_{k} \in \operatorname{spec} \sqrt{-\Delta}} e^{i R_{k} \xi} .
$$

In contrast with the case when all closed geodesics are isolated and $\chi(\xi)$ has singularities of the homogeneity order -1 at the points of the geodesic spectrum of 
the riemannian manifold (see $[9,10]$ ), in our case when closed geodesics form the families filling up the invariant tori, $\chi(\xi)$ has stronger singularities at the points of the geodesic spectrum, of the homogeneity order $-3 / 2$.

Theorem 3.3. Let $Q$ be a Liouville surface which satisfies the assumptions of Theorem 3.1. Consider the tempered distributions

$$
\begin{aligned}
d(x)= & \sum_{R_{k} \in \operatorname{spec} \sqrt{-\Delta}} \delta\left(x-R_{k}\right)-\frac{\operatorname{Area} Q}{2 \pi} x+-x_{+}^{1 / 2}\left(2 \pi^{3}\right)^{-1 / 2} \sum_{g \in G}|g|^{-3 / 2} \chi(g)^{-1 / 2} \\
& \left.\times \cos \left(|g| x-\frac{\pi}{2} \text { ind } g-\frac{\pi}{4} \sigma(g)\right)\right)
\end{aligned}
$$

and

$$
\begin{aligned}
\widehat{d}(\xi)= & \sum_{R_{k} \in \operatorname{spec} \sqrt{-\Delta}} e^{i R_{k} \xi}+\frac{\operatorname{Area} Q}{2 \pi}(\xi+i 0)^{-2}-\left(2 \pi^{3}\right)^{-1 / 2} \sum_{g \in G \pm} \sum_{|g|^{-1 / 2}} \chi(g)^{-1 / 2} \\
& \times e^{ \pm \frac{\pi i}{4}(2 \text { ind } g+\sigma(g))} v(\xi \mp|g|) .
\end{aligned}
$$

If a $C^{3}$-function $\varphi(t)$ satisfies the inequalities

$$
\left|\frac{d^{k} \varphi}{d t^{k}}\right| \leqq \operatorname{const}(k)\left(1+|t|^{2+k}\right)^{-1}, \quad k=0,1,2,3,
$$

then

$$
T^{-1} \int_{0}^{T} t^{-1 / 2}|(d * \varphi)|(t) d t \rightarrow 0 \quad \text { as } \quad T \rightarrow \infty
$$

and

$$
T^{-1} \int_{0}^{T} t^{-1 / 2}|\langle\widehat{d}, \exp (-i t \xi) \varphi\rangle| d t \rightarrow 0 \quad \text { as } \quad T \rightarrow \infty
$$

It is worthwhile to note that the general trace formula discussed in $[9,10]$ and the trace formula of Theorem 3.3 in a sense complement each other. Namely, to the local $L^{1}$ property of the function $\widehat{d}(\xi)$ in the general trace formula of $[9,10]$, the formula (3.13) adds some control of this function at infinity.

\section{Proofs}

As was shown in [15], the study of the spectrum of the Laplace-Beltrami operator on a Liouville surface can be reduced to the study of distribution of lattice points with respect to some particular domains dilated with some factor and then shifted. For the convenience of the reader we describe below this reduction proven in [15], and then we derive the desired theorems from the corresponding results for the lattice-point problem. It should be noted that the latter is of interest in itself.

Here we give a reduction of Theorem 3.1 to Theorems 2.1(a) and 11.1(i) of [15]. According to [15], the study of the function $N(x)$ can be reduced to the following lattice-point problem. Consider the curve $\Gamma$ on a plane given parametrically as

$$
x=\left(x_{1}(c), x_{2}(c)\right), \quad c_{4} \leqq c \leqq c_{1},
$$


where

$$
\begin{aligned}
& x_{1}(c)=F_{1}(c)=\int_{q_{1}: U_{1}\left(q_{1}\right)-c \geqq 0}\left(U_{1}\left(q_{1}\right)-c\right)^{1 / 2} d q_{1}, \\
& x_{2}(c)=F_{2}(c)=\int_{q_{2}: c-U_{2}\left(q_{2}\right) \geqq 0}\left(c-U_{2}\left(q_{2}\right)\right)^{1 / 2} d q_{2} .
\end{aligned}
$$

Observe that $x_{1}^{\prime}(c)<0$ and $x_{2}^{\prime}(c)>0$. In addition, $x_{1}\left(c_{1}\right)=x_{2}\left(c_{4}\right)=0$. This implies that $\Gamma$ is a star-like curve in the first quadrant so it can be written in the polar coordinates $\rho, \alpha$ as a graph of an single-valued function,

$$
\rho=G(\alpha), \quad 0 \leqq \alpha \leqq \pi / 2 .
$$

Define the angles $0=\alpha_{4}<\alpha_{3}<\alpha_{2}<\alpha_{1}=\pi / 2$ as solution of the equations

$$
\tan \alpha_{i}=\frac{F_{2}\left(c_{i}\right)}{F_{1}\left(c_{i}\right)}, \quad i=1,2,3,4
$$

and partition the first quadrant into three sectors $A_{1}, A_{2}, A_{3}$ with

$$
A_{i}=\left\{(\rho, \alpha): \alpha_{i+1} \leqq \alpha \leqq \alpha_{i}\right\}
$$

Theorem 4.1. ([15], Theorem 6.3). Let $Q \in Q^{r}, r \geqq 3$ be a Liouville surface. Then

(a) $G(\alpha) \in C^{1}\left(\left(0, \frac{1}{2} \pi\right)\right)$ and the tangent line to $\Gamma$ at the point with the angle coordinate $\alpha_{i}$ is parallel to the $x_{i}$-axis $(i=1,2)$.

(b) $G(\alpha) \in C^{r+1}\left(\left(0, \alpha_{1}\right) \cup\left(\alpha_{1}, \alpha_{2}\right) \cup\left(\alpha_{2}, \frac{1}{2} \pi\right)\right)$.

(c) For all $l(0 \leqq l \leqq r+1)$ there exist finite limits

$$
\begin{gathered}
\lim _{\alpha \rightarrow+0} \frac{d^{l} G}{d \alpha^{l}}(\alpha)=G^{(l)}(+0), \\
\lim _{\alpha \rightarrow \frac{1}{2} \pi-0} \frac{d^{l} G}{d \alpha^{l}}(\alpha)=G^{(l)}\left(\frac{1}{2} \pi-0\right) .
\end{gathered}
$$

In addition, $G^{(0)}(+0)=G(0)>0, G^{(0)}\left(\frac{1}{2} \pi-0\right)=G\left(\frac{1}{2} \pi\right)>0$ and $G^{(1)}(+0) \neq 0$, $G^{(1)}\left(\frac{1}{2} \pi-0\right) \neq 0$.

(d) In the vicinity of the critical angles $\alpha_{1}, \alpha_{2}$ derivatives of $G$ have the following asymptotics:

$$
\frac{d^{l} G}{d \alpha^{l}}(\alpha) \sim \frac{\operatorname{const}(i, l)}{\left(\alpha-\alpha_{i}\right)^{l-1}\left(\log \left|\alpha-\alpha_{l}\right|^{-1}\right)^{2}}, \quad \text { as } \quad \alpha \rightarrow \alpha_{i}, i=1,2,1 \leqq l \leqq r-1 .
$$

(e) The following inequalities hold for $0 \leqq \alpha \leqq \frac{1}{2} \pi$ :

$$
\begin{aligned}
& G(\alpha) \geqq \text { const }>0, \\
& \left|\frac{d G}{d \alpha}(\alpha)\right| \leqq \text { Const } .
\end{aligned}
$$


Denote by $D_{1}, D_{2}, D_{3}$ the finite sectorial domains cut off by the curve $\Gamma$ from the sectors $A_{1}, A_{2}, A_{3}$, respectively. Let $R D_{i}$ be the image of $D_{i}$ under the dilation with the factor $R$ with respect to the origin. Consider the lattices

$$
\begin{aligned}
& L_{1}=\left\{\left(\pi\left(m_{1}+(1 / 2)\right), 2 \pi m_{2}\right),\left(m_{1}, m_{2}\right) \in \mathbb{Z}^{2}\right\}, \\
& L_{2}=\left\{\left(2 \pi m_{1}, 2 \pi m_{2}\right),\left(m_{1}, m_{2}\right) \in \mathbb{Z}^{2}\right\}, \\
& L_{3}=\left\{\left(2 \pi m_{1}, \pi\left(m_{2}+(1 / 2)\right)\right),\left(m_{1}, m_{2}\right) \in \mathbb{Z}^{2}\right\},
\end{aligned}
$$

and define

$$
\begin{aligned}
& N_{i}(R)=\#\left\{L_{i} \cap R D_{i}\right\}, \quad i=1,2,3, \\
& N_{0}(R)=2 N_{1}(R)+4 N_{2}(R)+2 N_{3}(R) .
\end{aligned}
$$

Theorem 4.2. ([15]), Theorem 6.2). If a Liouville surface $Q \in \mathscr{Q}^{r}, r \geqq 5$, then the function

$$
\theta_{1}(R)=R^{-1 / 2}\left(N\left(R^{2}\right)-N_{0}(R)\right)
$$

is an almost periodic function equivalent to 0 in the Besicovitch class $B^{1}$, i.e.,

$$
\lim _{T \rightarrow \infty} \frac{1}{T} \int_{0}^{T}\left|\theta_{1}(R)\right| d R=0
$$

Denote $\Gamma_{i}=\Gamma \cap A_{i}$. Let

$$
\begin{aligned}
& D_{4}=\left\{\left(x_{1}, x_{2}\right) \in \mathbb{R}^{2}:\left(\pi x_{1}, 2 \pi\left|x_{2}\right|\right) \in D_{1}\right\}, \\
& D_{5}=\left\{\left(x_{1}, x_{2}\right) \in \mathbb{R}^{2}:\left(2 \pi\left|x_{1}\right|, 2 \pi\left|x_{2}\right|\right) \in D_{2}\right\}, \\
& D_{6}=\left\{\left(x_{1}, x_{2}\right) \in \mathbb{R}^{2}:\left(2 \pi\left|x_{1}\right|, \pi x_{2}\right) \in D_{3}\right\},
\end{aligned}
$$

and for a point $a \in \mathbb{R}^{2}$ and a domain $D \subset \mathbb{R}^{2}$, put

$$
\begin{gathered}
N(R, a, D)=\#\left\{\left(a+\mathbb{Z}^{2}\right) \cap R D\right\}, \\
n(R, a, D)=N(R, a, D)-R^{2} \text { Area } D .
\end{gathered}
$$

Obviously,

$$
N\left(R, a_{4}, D_{4}\right)=2 N_{1}(R), \quad N\left(R, a_{5}, D_{5}\right)=4 N_{2}(R), \quad N\left(R, a_{6}, D_{6}\right)=2 N_{3}(R),
$$

with

$$
\left.a_{4}=((1 / 2), 0), \quad a_{5}=(0,0), \quad a_{6}=(0,1 / 2)\right),
$$

hence

$$
N_{0}(R)=N\left(R, a_{4}, D_{4}\right)+N\left(R, a_{5}, D_{5}\right)+N\left(R, a_{6}, D_{6}\right)
$$

and by (4.5),

$$
\lim _{T \rightarrow \infty} \frac{1}{T} \int_{0}^{T} R^{-1 / 2}\left|N\left(R^{2}\right)-N\left(R, a_{4}, D_{4}\right)-N\left(R, a_{5}, D_{5}\right)-N\left(R, a_{6}, D_{6}\right)\right| d R=0 .
$$

By the Weyl law (see (1.5)),

$$
N\left(R^{2}\right)=\frac{\text { Area } Q}{4 \pi} R^{2}+n\left(R^{2}\right)
$$


with $n\left(R^{2}\right)=o\left(R^{2}\right)$, and it is easy to see that

$$
N\left(R, a_{i}, D_{i}\right)=\left(\text { Area } D_{i}\right) R^{2}+n\left(R, a_{i}, D_{i}\right), \quad i=4,5,6,
$$

with $n\left(R, a_{i}, D_{i}\right)=o\left(R^{2}\right)$. Hence (4.7) implies

$$
\frac{\text { Area } Q}{4 \pi}=\operatorname{Area} D_{4}+\operatorname{Area} D_{5}+\text { Area } D_{6}
$$

and

$$
\lim _{T \rightarrow \infty} \frac{1}{T} \int_{0}^{T} R^{-1 / 2}\left|n\left(R^{2}\right)-n\left(R, a_{4}, D_{4}\right)-n\left(R, a_{5}, D_{5}\right)-n\left(R, a_{6}, D_{6}\right)\right| d R=0 .
$$

This equation shows that we have reduced Theorem 3.1 to a problem of number theory about the behaviour of the number of lattice points inside a dilated domain.

Let $D_{0}$ be a star-like domain given in the polar coordinates by the inequalities

$$
0 \leqq \rho \leqq G_{0}(\beta), \beta_{1} \leqq \beta \leqq \beta_{2}
$$

and denote by $\Gamma_{0}$ a part of its boundary given by equation:

$$
\rho=G_{0}(\beta), \beta_{1} \leqq \beta \leqq \beta_{2} .
$$

Assume that:

(A1) $\Gamma_{0} \in C^{1}\left(\left[\beta_{1}, \beta_{2}\right]\right) \cap P C^{5}\left(\left(\beta_{1}, \beta_{2}\right)\right)$ where $P C^{r}$ denotes the space of piecewise $C^{r}$-functions, and for $\beta_{1} \leqq \beta \leqq \beta_{2}$,

$$
0<c \leqq G_{0}(\beta) \leqq C, \quad\left|G^{\prime}(\beta)\right| \leqq C
$$

(A2) The curvature $\varkappa_{0}(\beta)$ of $\Gamma_{0}$ has only finitely many zeroes and each zero has the first order. More precisely, if $x_{0}\left(\beta_{0}\right)=0$ then

$$
\varkappa_{0}(\beta)=\left(\beta-\beta_{0}\right) \varkappa_{1}(\beta) \text { with } \varkappa_{1}\left(\beta_{0}\right) \neq 0 .
$$

(A3) There are only finitely many points on $\Gamma_{0}$ with infinite curvature. If $\beta^{*}$ is such a point then in the vicinity of $\beta^{*}$ the function $\chi_{0}$ and its derivatives have the following asymptotics:

$$
\frac{d^{k} \varkappa_{0}(\beta)}{d \beta^{k}} \sim \frac{C\left(\beta^{*}, k\right)}{\left(\beta-\beta^{*}\right)^{1+k}\left(\log \left|\beta-\beta^{*}\right|^{-1}\right)^{2}}, k=0,1,2,3 .
$$

Here $C\left(\beta^{*}, k\right)$ are nonvanishing real numbers depending on the critical point $\beta^{*}$.

Denote by $V$ the set of all points of $\Gamma_{0}$ with infinite or zero curvature and include also in $V$ the endpoints of $\Gamma_{0}$ if it is not closed.

(A4) If $V \neq \emptyset$ then for each point from $V$ with finite curvature the directions of its radius-vector and the tangent line to $\Gamma_{0}$ at this point are diophantine (i.e. the tangents of the slope angles are diophantine). For points where the curvature is infinite the directions of the radius vectors of these points are diophantine and the directions of the tangent lines at these points are either diophantine or rational. 
For a given curve $\Gamma_{0}$ define the function $S_{0}(\eta)$, which maps a vector $\eta \in \mathbb{R}^{2} \backslash\{0\}$ onto the set of all points $x$ on $\Gamma_{0}$ for which the vector of outer normal $n(x)$ to $D_{0}$ has the same direction as $\eta$, i.e.,

$$
S_{0}(\eta)=\left\{x \in \Gamma_{0}: n(x)=\frac{\eta}{|\eta|}\right\} .
$$

For some $\eta$ the set $S_{0}(\eta)$ can be empty. Note that the assumptions A1, A2 imply that the curve $\Gamma_{0}$ consists of finitely many concave and convex arcs. This in turn implies $S_{0}(\eta)$ to be a finite set whose cardinality is uniformly bounded from above.

Define

$$
E=\left\{n=\left(n_{1}, n_{2}\right) \in \mathbb{N}: n_{1}, n_{2} \text { are coprime }\right\} \cup\{(1,0),(0,1)\},
$$

and

$$
\Lambda=\cup_{\eta \in E}\left\{\langle x, \eta\rangle \mid x \in S_{0}(\eta)\right\}
$$

A(5) The elements of $\Lambda$ are linearly independent over $\mathbb{Z}$.

Theorem 4.3. ([15]), Theorem 11.1 (i), Lemmas 13.1-13.8). If the domain $D_{0}$ satisfies the assumptions A1-A4 then for any $a \in \mathbb{R}^{2}$ the function $\theta_{0}(R)=$ $R^{-1 / 2} n\left(D_{0}, a, R\right)$ is an almost periodic function of the Besicovitch class $B^{2}$. The Fourier series of $\theta_{0}$ with respect to $B^{2}$ has the form:

$$
\begin{aligned}
\theta_{0}(R)= & \pi^{-1} \sum_{m \in \mathbb{Z}^{2} \backslash 0} \sum_{x \in S_{0}(m)}|m|^{-3 / 2}\left|\kappa_{0}(x)\right|^{-1 / 2} \\
& \times \sin \left(2 \pi R\langle x, m\rangle-2 \pi\langle a, m\rangle-\frac{1}{4} \pi \operatorname{sign} \varkappa_{0}(x)\right) .
\end{aligned}
$$

In addition, for any $T$ and $K$ sufficiently large and $\beta>0$

$$
T^{-1} \int_{0}^{T}\left|\theta_{0}(R)-P_{K}(R)\right|^{2} d R \leqq \operatorname{const}(\beta)\left(T^{-\frac{1}{2}+\beta}+K^{-\frac{1}{6}+\beta}+K^{\frac{5}{12}} T^{-12}\right),
$$

where

$$
\begin{aligned}
P_{K}(R)= & \pi^{-1} \sum_{\substack{m \in \mathbb{Z}^{2} \backslash 0 \\
|m| \leqq K \\
x \in S_{0}(m)}}|m|^{-3 / 2}\left|\varkappa_{0}(x)\right|^{-1 / 2} \\
& \times \sin \left(2 \pi R\langle x, m\rangle-2 \pi\langle a, m\rangle-\frac{1}{4} \pi \operatorname{sign} \varkappa_{0}(x)\right) .
\end{aligned}
$$

Theorem 4.4. ([15], Theorem 11.1 (ii)). If in addition to the assumptions of Theorem 4.3 the condition $\mathrm{A} 5$ is also fulfilled, then the limit distribution $\mu(d y)$ of $\theta_{0}(R)$ does not depend on $a \in \mathbb{R}^{2}$ and has a density $p(y)$ with respect to the Lebesgue measure. The density $p(y)$ is an entire function of its argument and satisfies the following inequalities on the real line: $\forall \varepsilon>0 \exists y(\varepsilon)>0$ such that

$$
p(y) \leqq \exp \left(-|y|^{\frac{16}{9}-\varepsilon}\right) \quad \text { when } \quad|y| \geqq y(\varepsilon) .
$$

For a finite collection of domains $B_{1}, B_{2}, \ldots B_{l}$, consider the linear combination

$$
n_{s}(R)=\sum s_{i} n\left(R, a_{i}, B_{i}\right) \text {, }
$$


where $s_{i}, i=1, \ldots, l$, are arbitrary real numbers. Denote by $\Lambda_{i}$ the set defined by (4.13) with $E=B_{i}$.

Theorem 4.5. ([15]), Corollary 11.2). If the assumptions A1-A4 are satisfied for each domain $B_{i}$ then the function $R^{-1 / 2} n_{s}(R)$ belongs to the Besicovitch class $B^{2}$ of almost periodic functions. In addition, if the assumption A5 holds for the set $\Lambda=\cup \Lambda_{i}$ then the density $p(y)$ of the limit distribution of $R^{-1 / 2} n_{s}(R)$ posesses all the properties formulated in Theorem 4.4 .

In the proof of Theorem 3.2 we use an improvement of the estimate (4.17) which will be proven in Sect. 5 below. For an exact formulation of the improved estimate we need some definitions.

Define

$$
\varphi(\lambda)=\int_{0}^{1} \exp (i \lambda B(t)) d t,
$$

where

$$
B(t)=\sum_{m=1}^{\infty} m^{-3 / 2} \sin (2 \pi m t-(\pi / 4))
$$

and

$$
A(\lambda)=(4 / 3) \int_{0}^{\infty} x^{-7 / 3} \log \varphi(\lambda x) d x
$$

It follows from (4.18) that $\varphi(i y)$ is a positive real-analytic function of $y \in \mathbb{R}$ with $\varphi(0)=1$ and $\varphi^{\prime}(0)=0$. In addition, $\varphi(i y)$ has exponential asymptotics as $t \rightarrow \pm \infty$. This implies that the RHS of (4.20) is well-defined for $\lambda$ pure imaginary and

$$
A(i v)= \begin{cases}A_{+} v^{4 / 3} & \text { if } \quad v>0, \\ A_{-}(-v)^{4 / 3} & \text { if } \quad v<0,\end{cases}
$$

with $A_{ \pm}=A( \pm i)$. We will show in Sect. 6 below that there exists $\delta>0$ such that the RHS of (4.20) is well-defined for all $\lambda$ in the sector

$$
S_{\delta}=\{\lambda \in \mathbb{C} \backslash\{0\}:|\operatorname{Re} \lambda| \leqq \delta|\operatorname{Im} \lambda|\},
$$

and

$$
A\left(i e^{i \theta} v\right)=A_{ \pm}\left( \pm e^{i \theta} v\right)^{4 / 3}
$$

for all $|\theta| \leqq \delta$ and $\pm v>0$.

Define

$$
\Phi(\lambda)=\prod_{m \in E} \prod_{x \in S_{0}(m)} \varphi\left(\pi^{-1} \rho(m, x) \lambda\right),
$$

where $E, S_{0}(m)$ and $\varphi(\lambda)$ are defined in the formulas (4.12), (4.11) and (4.18), respectively,

$$
\rho(m, x)=|m|^{-3 / 2} \operatorname{sign}(\varkappa(x))|x(x)|^{-1 / 2}
$$

and $x(x)$ is the curvature at the point $x$.

Theorem 4.6. Let all the conditions of Theorem 4.5 be satisfied. Then the characteristic function $\Phi_{s}(\lambda)$ of the limit distribution of $R^{-1 / 2} n_{s}(R)$ is equal to the product

$$
\Phi_{s}(\lambda)=\Phi^{(1)}\left(s_{1} \lambda\right) \cdots \Phi^{(l)}\left(s_{l} \lambda\right)
$$


where the function $\Phi^{(i)}(\lambda)(1 \leqq i \leqq l)$ is defined by (4.23) with the help of the domain $B_{i}$, and the density $p(t)$ of this limit distribution is an entire function of $t$ such that for real $t$,

$$
-t^{-4} \log p(t) \rightarrow C_{ \pm}>0 \text { as } t \rightarrow \infty
$$

with

$$
C_{ \pm}=8 \pi^{4}\left(\sum_{j=1}^{l} \int_{\partial B_{j}} A\left( \pm i s_{j} \operatorname{sgn} \varkappa_{j}(x)\right)\left|\varkappa_{j}(x)\right|^{1 / 3} d l_{j}(x)\right)^{-3} .
$$

Here dl stands for the differential of the length of arc along the curve.

Proof of Theorem 3.1. Theorems 4.1, 4.2, 4.3, and 4.5 expressed in terms of the geodesic flow on Liouville surface give the desired result immediately.

Let

$$
\begin{aligned}
& \Gamma_{4}=\left\{\left(x_{1}, x_{2}\right) \in \mathbb{R}^{2}:\left(\pi x_{1}, 2 \pi\left|x_{2}\right|\right) \in \Gamma_{1}\right\}, \\
& \Gamma_{5}=\left\{\left(x_{1}, x_{2}\right) \in \mathbb{R}^{2}:\left(2 \pi\left|x_{1}\right|, 2 \pi\left|x_{2}\right|\right) \in \Gamma_{2}\right\}, \\
& \Gamma_{6}=\left\{\left(x_{1}, x_{2}\right) \in \mathbb{R}^{2}:\left(2 \pi\left|x_{1}\right|, \pi x_{2}\right) \in \Gamma_{3}\right\},
\end{aligned}
$$

so that $\Gamma_{i}$ is the curvilinear part of the boundary of $D_{i}$ (cf. (4.6)). Denote by $S_{i}(\eta), i=4,5,6$, the function $S_{0}(\eta)$ constructed as described above with the help of the curve $\Gamma_{i}$ instead of $\Gamma_{0}$ (see the paragraph before (4.12)). According to Theorems 4.2, 4.5 the Fourier series of $\theta(R)$ is

$$
\begin{aligned}
\theta(R)= & \pi^{-1} \sum_{i=4,5,6} \sum_{\substack{m \in \mathbb{Z}^{2}\left\{\{0\} \\
x \in S_{i}(m)\right.}}|m|^{-3 / 2}\left|\varkappa_{i}(x)\right|^{-1 / 2} \\
& \times \sin \left(2 \pi\langle m, x\rangle R-2 \pi\left\langle m, a_{i}\right\rangle-(\pi / 4) \operatorname{sign} \varkappa_{i}(x)\right),
\end{aligned}
$$

where $|m|=\left(m_{1}^{2}+m_{2}^{2}\right)^{1 / 2},\langle m, x\rangle=m_{1} x_{1}+m_{2} x_{2}$ and $x_{i}(x)$ is the curvature of $\Gamma_{i}$ at the point $x \in \Gamma_{i}$.

We can establish a one-to-one correspondence between the set of invariant tori $w \in W$ filling up the unit cotangent bundle and the set

$$
\Gamma_{*}=\Gamma_{4} \cup \Gamma_{5} \cup \Gamma_{6} .
$$

Namely, let an invariant torus $w \in W$ correspond to the point $x(w)=$ $(2 \pi)^{-1}\left(I_{1}, I_{2}\right)$, where $I_{1}, I_{2}$ are the values of the action variables on $w$ (see (2.4), (2.5)). Comparing (2.4), (2.5) with (4.1), (4.26) we see that $x(w) \in \Gamma_{*}$. Observe that $E=1 / 2$ on any $w \in W$ hence by (2.7) $I_{i}=f_{i}$. Therefore, (2.7), (2.8) imply that the vector $\left(\omega_{1}, \omega_{2}\right)$ is collinear to the vector of the outer normal to $\Gamma_{*}$ at the point $x(w)$. This allows us to establish a one-to-one correspondence between the set $G$ of families of closed geodesics on $Q$ and summands in (4.27). Namely, every $g \in G$ is characterized by an invariant torus $w(g) \in W$ where it lives and by the rotation numbers $n_{1}(g), n_{2}(g)$. So we can define the map

$$
g \rightarrow\left(m=\left(n_{1}(g), n_{2}(g)\right), x=x(w(g))\right),
$$

which is the one-to-one correspondence between $G$ and the summands in (4.27). 
Distribution of Energy Levels of Quantum Free Particle

Since the equation of $\Gamma_{*}$ is $x(c)=(2 \pi)^{-1}\left(f_{1}(c), f_{2}(c)\right)$ the curvature $\chi_{i}(x)$ is

$$
x_{i}(x)=2 \pi\left(f_{1}^{\prime} f_{2}^{\prime \prime}-f_{2}^{\prime} f_{1}^{\prime \prime}\right)\left(f_{1}^{\prime 2}+f_{2}^{\prime 2}\right)^{-3 / 2} .
$$

By (2.18),

$$
|m|^{2}=n_{1}^{2}+n_{2}^{2}=|g|^{2}\left(\omega_{1}^{2}+\omega_{2}^{2}\right)=|g|^{2}\left(\xi_{1}^{2}+\xi_{2}^{2}\right)
$$

hence

$$
|m|^{3} \varkappa_{i}(x)=|g|^{3}\left(\xi_{1}^{2}+\xi_{2}^{2}\right)^{3 / 2} 2 \pi\left(f_{1}^{\prime} f_{2}^{\prime \prime}-f_{2}^{\prime} f_{1}^{\prime \prime}\right)\left(f_{1}^{\prime 2}+f_{2}^{\prime 2}\right)^{-3 / 2},
$$

and by $(2.15)$,

$$
|m|^{3} k_{i}(x)=2 \pi|g|^{3} \operatorname{det}\left(d^{2} H\right)((1 / 2), c) .
$$

Also, (2.18) implies

$$
2 \pi\langle m, x\rangle=n_{1} f_{1}+n_{2} f_{2}=|g|
$$

By (4.28),

$$
\operatorname{sgn}\left(d^{2} H\right)((1 / 2), c)=\operatorname{sgn} \varkappa_{i}(x),
$$

and the number $4\left\langle m, a_{i}\right\rangle$ mod 4 is the Maslov index of $g \in G$. Thus the formula (3.3) follows from (4.27).

In addition, the formulae (2.3)-(2.5), (2.8), (2.14), (2.15) and Theorem 4.1 show that the conditions of Theorem 3.1 coincide with the conditions of Theorem 4.3 formulated in terms of the geodesic flow on $Q$ which completes the proof of Theorem 3.1.

Proof of Theorem 3.2. Invariant tori $w \in W_{+}$of the unit cotangent bundle with non-negative frequencies correspond to the points of

$$
\Gamma_{0}=\Gamma_{*} \cap\left\{x=\left(x_{1}, x_{2}\right) \in \mathbb{R}^{2}: x_{1}, x_{2} \geqq 0\right\} .
$$

We define the multiplicity function $h(x)$ on $\Gamma^{0}$ as

$$
h(x)=\left\{\begin{array}{lll}
2 & \text { if } & x \in \Gamma_{4} \\
4 & \text { if } & x \in \Gamma_{5} \\
2 & \text { if } & x \in \Gamma_{6}
\end{array}\right.
$$

Consider the set

$$
E=\left\{n=\left(n_{1}, n_{2}\right) \in \mathbb{N}: n_{1}, n_{2} \text { are relatively prime }\right\} \cup\{(1,0),(0,1)\} .
$$

Then

$$
\Omega=\left\{|g|, g \in G_{0}\right\}=\bigcup_{m \in E}\left\{2 \pi\langle m, x\rangle, x \in S_{0}(m)\right\} .
$$

Theorem 3.2 follows directly from Theorems 4.5, 4.6 in view of the formula (4.28).

Proof of Theorem 3.3. To prove (3.12) let us first observe that if $\varphi(t)$ satisfies (3.11) then the series

$$
\begin{aligned}
& \sum_{g \in G}|g|^{-3 / 2} \varkappa(g)^{-1 / 2} \int_{0}^{\infty} \varphi^{\prime}(x-y) y^{1 / 2} \sin \left(|g| y-\frac{\pi}{2} \operatorname{ind} g-\frac{\pi}{4} \sigma(g)\right) d y \\
& =\sum_{g \in G}|g|^{-5 / 2} \varkappa(g)^{-1 / 2} \int_{0}^{\infty}\left[\varphi^{\prime}(x-y) y^{1 / 2}\right]^{\prime} \cos \left(|g| y-\frac{\pi}{2} \operatorname{ind} g-\frac{\pi}{4} \sigma(g)\right) d y
\end{aligned}
$$


is absolutely convergent because the sum over $g$ is basically a two-dimensional lattice sum and $\sum_{g}|g|^{-5 / 2}<\infty$. This implies that if we define the tempered distribution $d_{K}(x)$ as

$$
\begin{aligned}
d_{K}(x)= & \sum_{R_{k} \in \operatorname{spec} \sqrt{-\Delta}} \delta\left(x-R_{k}\right)-\frac{\text { Area } Q}{2 \pi} x_{+}-\left(2 \pi^{3}\right)^{-1 / 2} \sum_{g \in G:|g| \leqq K}|g|^{-3 / 2} \\
& \times x(g)^{-1 / 2}\left[x_{+}^{1 / 2} \sin \left(|g| x-\frac{\pi}{2} \text { ind }-\frac{\pi}{4} \sigma(g)\right)\right]^{\prime},
\end{aligned}
$$

then

$$
\lim _{K \rightarrow \infty} \lim _{T \rightarrow \infty} \frac{1}{T} \int_{0}^{T} t^{-1 / 2}\left|\left(d-d_{K}\right) * \varphi(t)\right| d t=0 .
$$

On the other hand,

$$
d_{K} * \varphi(t)=\varepsilon_{K} * \varphi^{\prime}(t)
$$

where

$$
\begin{aligned}
\varepsilon_{K}(t)= & N\left(t^{2}\right)-\frac{\text { Area } Q}{4 \pi} t_{+}^{2}-\left(2 \pi^{3}\right)^{-1 / 2} \sum_{g \in G:|g| \leqq K}|g|^{-3 / 2} \varkappa(g)^{-1 / 2} \\
& \times t_{+}^{1 / 2} \sin \left(|g| x-\frac{\pi}{2} \text { ind }-\frac{\pi}{4} \sigma(g)\right),
\end{aligned}
$$

and by Theorem 3.1

$$
\lim _{K \rightarrow \infty} \lim _{T \rightarrow \infty} \frac{1}{T} \int_{0}^{T} t^{-1 / 2}\left|\varepsilon_{K}(t)\right| d t=0
$$

hence

$$
\lim _{K \rightarrow \infty} \lim _{T \rightarrow \infty} \frac{1}{T} \int_{0}^{T} t^{-1 / 2}\left|\varepsilon_{K} * \varphi^{\prime}(t)\right| d t=0 .
$$

From (4.30)-(4.32) we obtain (3.12). Since $\widehat{d}$ is the Fourier transform of $d,(3.13)$ follows from (3.12). Theorem 3.3 is proved.

\section{Proof of Theorem 4.6}

Proof of Theorem 4.6. We consider a particular case when $l=1, s_{1}=1$ and $D=D_{1}$ is convex. The proof for the case of several convex and concave arcs goes mutatis mutandis.

Using the inverse Fourier transform we obtain

$$
p(t)=(2 \pi)^{-1} \int_{-\infty}^{\infty} \Phi(\lambda) e^{-i t \lambda} d \lambda .
$$

Lemma 5.1. (see [15], Lemma 15.1). If the conditions of Theorem 4.4 are satisfied then the function $\Phi(\lambda)$ is an entire function of $\lambda=\lambda_{1}+i \lambda_{2}$ satisfying the estimation

$$
|\Phi(\lambda)| \leqq \exp \left(-C_{1}\left|\lambda_{1}\right|^{2}|\lambda|^{-2 / 3}+C_{2}\left|\lambda_{2}\right||\lambda|^{3 / 4}\right) \quad \text { as }|\lambda| \geqq 1
$$

where $C_{1}, C_{2}$ are some positive constants. 
By Cauchy's theorem and with the help of Lemma 5.1 we can shift the axis of integration in (5.1),

$$
p(t)=(2 \pi)^{-1} \int_{-\infty}^{\infty} \Phi(\mu+i v) \exp (-i t(\mu+i v)) d \mu
$$

We evaluate the asymptotics of $p(t)$ as $t \rightarrow \pm \infty$ with the help of the saddle-point method. To this end we put

$$
\Psi(\lambda)=\log \Phi(\lambda)=\sum_{m \in E} \psi\left(\pi^{-1} \rho(m) \lambda\right)
$$

where $\psi(\lambda)=\log \varphi(\lambda)$ and $\rho(m)=\rho\left(m, S_{0}(m)\right.$ ) (recall that we assume that $S_{0}(m)$ consists of one point). Due to Lemma 7.5 formulated in Sect. 7 below, there exists a $\delta>0$ so that for $\lambda$ such that $\left|\lambda_{1}\right| \leqq \delta\left|\lambda_{2}\right|$, the function $\psi(\lambda)$ is well defined and analytic. Let us choose $\delta>0$ so that all the statements of Sect. 7 are valid and rewrite (5.2) as

$$
\begin{aligned}
p(t)= & (2 \pi)^{-1} \int_{|\mu| \leqq \delta|v|} \exp (\Psi(\mu+i v)-i t(\mu+i v)) d \mu \\
& +(2 \pi)^{-1} \int_{|\mu| \geqq \delta|v|} \Phi(\mu+i v) \exp (-i t(\mu+i v)) d \mu \\
= & I_{1}(\delta)+I_{2}(\delta) .
\end{aligned}
$$

Lemma 5.2. If $\lambda \in S_{\delta}=\{z \in \mathbb{C} \backslash\{0\}:|\operatorname{Re} z| \leqq \delta|\operatorname{Im} z|\}$, then

$$
\Psi(\lambda)=(\varkappa / 2) A\left(\pi^{-1} \lambda\right)(1+o(1)) \text { as }|\lambda| \rightarrow \infty,
$$

uniformly in arg $\lambda$. Here

$$
\varkappa=\varkappa(\Gamma)=\int_{\Gamma}|\chi(x)|^{1 / 3} d l(x)
$$

is the affine length of $\Gamma$, the curvilinear part of $\partial D$.

Corollary 5.3. If $\lambda \in S_{\delta}$ then

$$
\begin{aligned}
& \Psi^{\prime}(\lambda)=(4 / 3)(\pi \lambda)^{-1} \varkappa A\left(\pi^{-1} \lambda\right)(1+o(1)), \quad \text { as }|\lambda| \rightarrow \infty, \\
& \Psi^{\prime \prime}(\lambda)=(4 / 9)(\pi \lambda)^{-2} \varkappa A\left(\pi^{-1} \lambda\right)(1+o(1)),
\end{aligned}
$$

uniformly in arg $\lambda$.

Corollary 5.3 is an obvious consequence of the Cauchy integration formula and Lemma 5.2, and we leave it without proof.

Let us apply the saddle-point method to the integral $I_{1}(\delta)$. If $\delta$ is chosen small enough then Lemma 7.5 below shows that the function $\Psi(\lambda)-i t \lambda$ has in $S_{\delta}$ for large $|t|$ a unique critical point $\lambda_{c}(t)=\mu_{c}(t)+i v_{c}(t)$ with $\mu_{c}(t)=0$. Taking $v=v_{c}(t)$ in (5.2) we obtain, with the help of the saddle-point method, the following lemma:

\section{Lemma 5.4.}

$$
\log I_{1}(\delta)=-8 \pi^{4}\left(\varkappa A_{ \pm}\right)^{-3} t^{4}(1+o(1))
$$


The following lemma completes the proof of Theorem 4.6:

\section{Lemma 5.5.}

$$
I_{2}(\delta)=o\left(I_{1}(\delta)\right), \text { as } t \rightarrow \pm \infty
$$

\section{Proof of Technical Lemmas}

Proof of Lemma 5.2. Introduce the set of measures $\mu_{R}, R>0$, in the first quadrant,

$$
\mu_{R}=\left(\pi^{2} / 6\right) R^{-2} \sum_{m \in E} \delta\left(x-R^{-2} m\right) .
$$

The family $\mu_{R}$ weakly converges to the Lebesgue measure as $R \rightarrow \infty$ so that for any continuous function $g$ with compact support,

$$
\lim _{R \rightarrow \infty} R^{-2} \sum_{m \in E} g\left(R^{-1} m\right)=\left(6 / \pi^{2}\right) \int_{0}^{\infty} \int_{0}^{\infty} g(x, y) d x d y
$$

Consider a sequence of $C^{\infty}$ functions $\zeta_{n}(\lambda)$ equal 1 inside the disk $|\lambda| \leqq n$ and having the support in the disk $|\lambda| \leqq 2 n$. Applying (6.2) to the sums

$$
\Psi_{n}(\lambda)=\sum_{m \in E} \psi\left(\pi^{-1} \rho(m) \lambda\right) \zeta_{n}(\lambda)
$$

and letting $n \rightarrow \infty$ for $\lambda \in S_{\delta}$, we get uniformly in $\arg \lambda$,

$$
\sum_{m \in E} \Psi\left(\pi^{-1} \rho(m) \lambda\right)=\pi^{-4 / 3}|\lambda|^{4 / 3} \int_{0}^{\infty} \int_{0}^{\infty} \Psi(\rho(m) \exp (i \arg \lambda)) d m(1+o(1)) .
$$

Recall that the geometrical meaning of the vector $m$ is that it is a normal vector to $\Gamma$. Since $\Gamma$ is a convex curve it can be smoothly parametrized by the angle $\beta$ between the direction of the normal vector to $\Gamma$ and polar axis. Then we can split the variables of integration in (6.3):

$$
\begin{aligned}
|\lambda|^{4 / 3} \int_{0}^{\infty} \int_{0}^{\infty} \Psi & (\rho(m) \exp (i \arg \lambda)) d m \\
& =|\lambda|^{4 / 3} \int_{0}^{\infty} \int_{\Gamma} \Psi\left(\exp (i \arg \lambda) r^{-3 / 2} \varkappa(\beta)^{-1 / 2}\right) r d \beta d r \\
& =\frac{2}{3}|\lambda|^{4 / 3} \int_{0}^{\infty} \int_{\Gamma} \Psi\left(\exp (i \arg \lambda) s \varkappa(\beta)^{-1 / 2}\right) s^{-7 / 3} d \beta d s \\
& \left.=\frac{2}{3} \int_{0}^{\infty} \int_{\Gamma} \Psi(\lambda t) t^{-7 / 3} \chi(\beta)^{2 / 3}\right) d \beta d t .
\end{aligned}
$$

We changed in (6.4) the variables by putting $r^{-3 / 2}=s$ and $s=\chi(\beta)^{1 / 2}|\lambda| t$. Thus substituting the integration with respect to the arclength parametrization for the integration with respect to $\beta$ finally we get

$$
\begin{aligned}
\sum_{m \in E} \Psi\left(\pi^{-1} \rho(m) \lambda\right) & =(2 / 3) \varkappa \int_{0}^{\infty} \Psi\left(\pi^{-1} \lambda y\right) y^{-7 / 3} d y(1+o(1)) \\
& =(\varkappa / 2) A\left(\pi^{-1} \lambda\right)(1+o(1)) .
\end{aligned}
$$

Lemma 5.2 is proved. 
Proof of Lemma 5.4. Corollary 5.3 shows that $v_{c}(t)$ is the unique solution of the equation

$$
\Psi^{\prime}\left(i v_{c}(t)\right)-i t=0
$$

Hence using (4.21) we obtain

$$
v_{c}(t)=-\left(\frac{2}{3} x A_{ \pm}\right)^{-3} \pi^{4} t^{3}(1+o(1)), \quad t \rightarrow \pm \infty .
$$

Thus by $(4.20)$,

$$
\Psi\left(i v_{c}(t)\right)+t v_{c}(t)=-\left(\pi^{4} / 4\right)\left((2 / 3) \varkappa A_{ \pm}\right)^{-3} t^{4}(1+o(1)) \quad t \rightarrow \pm \infty .
$$

In addition, by (5.6)

$$
\Psi^{\prime \prime}\left(i v_{c}(t)\right)=C_{ \pm} t^{-6}(1+o(1)), \quad t \rightarrow \pm \infty,
$$

with some $C_{ \pm}>0$. From (6.7), (6.8) with the help of the saddle-point method we get that as $t \rightarrow \pm \infty$,

$$
\begin{aligned}
\log I_{1}(\delta)= & \log \left[(2 \pi)^{-1} \exp \left(\Psi\left(i v_{c}(t)\right)+t v_{c}(t)-\frac{1}{4} \pi \operatorname{sign} \Psi^{\prime \prime}\left(i v_{c}(t)\right)\right)\right. \\
& \left.\times\left|\Psi^{\prime \prime}\left(i v_{c}(t)\right)\right|^{-1 / 2}\right](1+o(1))=-\left(\pi^{4} / 4\right)\left((2 / 3) \varkappa A_{ \pm}\right)^{-3} t^{4}(1+o(1)) .
\end{aligned}
$$

Lemma 5.4 is proved.

Proof of Lemma 5.5. Fix a large number $N$. Then from Lemmas 7.2, 7.4 and formulae (6.7), (6.8) we have for large $|t|$,

$$
\begin{aligned}
\frac{\left|I_{2}(\delta)\right|}{\left|I_{1}(\delta)\right|} & \leqq c t^{4} \int_{|\mu|>\delta\left|v_{c}(t)\right|}\left|\frac{\Phi\left(\mu+i v_{c}(t)\right)}{\Phi\left(i v_{c}(t)\right)}\right| d \mu \\
& \leqq c t^{4} \int_{|\mu|>\delta\left|v_{c}(t)\right|} \prod_{m \in E} \prod_{x \in S(m)}\left|\frac{\varphi\left(\pi^{-1} \rho(m)\left(\mu+i v_{c}(t)\right)\right)}{\varphi\left(\pi^{-1} \rho(m)\left(i v_{c}(t)\right)\right)}\right| d \mu .
\end{aligned}
$$

If $|\lambda| \geqq N,|\operatorname{Re} \lambda| \geqq \delta|\operatorname{Im} \lambda|$ and $N$ is large enough then due to Corollary 7.5 ,

$$
\left|\frac{\varphi(\lambda)}{\varphi(\operatorname{Re} \lambda)}\right| \leqq\left(\frac{|\operatorname{Re} \lambda|}{|\lambda|}\right)^{1 / 2}(1+(\delta / 2))
$$

In addition, if $|t|$ is large then the number of $m \in E$ for which $S(m) \neq \emptyset$ and $\left|\pi^{-1} \rho(m)\left(\mu+i v_{c}(t)\right)\right| \geqq 1$ is bounded from below by $c t^{2} N^{-2 / 3}$. Hence

$$
\left|\frac{\Phi\left(\mu+i v_{c}(t)\right)}{\Phi\left(i v_{c}(t)\right)}\right| \leqq\left[(1+(\delta / 2))\left(1+\left|\frac{\mu}{v_{c}(t)}\right|\right)^{-1}\right]^{c N^{-2 / 3} t^{2}}
$$


and

$$
\begin{aligned}
\frac{\left|I_{2}(\delta)\right|}{\left|I_{1}(\delta)\right|} & \leqq c_{0} t^{4} \int_{\delta\left|v_{c}(t)\right|}^{\infty}\left[(1+(\delta / 2))\left(1+\left|\frac{\mu}{v_{c}(t)}\right|\right)^{-1}\right]^{c N^{-2 / 3} t^{2}} d \mu \\
& \leqq c_{1} t^{7}(1-(\delta / 2))^{c N^{-2 / 3} t^{2}} \rightarrow 0
\end{aligned}
$$

as $|t| \rightarrow \infty$. Lemma 5.5 is proved.

\section{Properties of Auxiliary Functions}

Define the functions

$$
\begin{aligned}
& b(x)=\sum_{m=1}^{\infty} m^{-3 / 2} \exp (2 \pi i m x), \\
& B(x)=\sum_{m=1}^{\infty} m^{-3 / 2} \sin (2 \pi m x-(\pi / 4)),
\end{aligned}
$$

which are continuous periodic functions of period 1. In addition, $b(x)$ can be extended to the upper complex half-plane as a bounded periodic analytic function of period 1. Remark that

$$
B(x)=\operatorname{Im}\left(\frac{1-i}{\sqrt{2}} b(x)\right) .
$$

Lemma 7.1. The function $B(x)$ is a continuous periodic function of period 1 with

$$
\int_{0}^{1} B(x) d x=0,
$$

and moreover $B(x)$ is a real analytic and strictly concave function on $(0,1)$, so that

$$
B^{\prime \prime}(x)<0, \quad \forall 0<x<1 .
$$

Also the function $B(x)$ has a unique maximum point $x_{\max }$ on the interval $(0,1)$ and

$$
B\left(x_{\max }\right)>0 \text {. }
$$

In addition, $B(x)$ is minimal at the point $x_{\mathrm{min}}=0$ and

$$
B(0)<0 .
$$

Near 0 the function $B(x)$ has the form

$$
B(x)=2 \pi(2 x)_{+}^{1 / 2}+b_{1}(x),
$$

where

$$
y_{+}^{1 / 2}=\left\{\begin{array}{lll}
y^{1 / 2} & \text { if } & y>0, \\
0 & \text { if } & y \leqq 0,
\end{array}\right.
$$

and $b_{1}(x)$ is analytic near 0 with $b_{1}^{\prime}(0)<0$. 
It is to be noted that by $(7.6), B(x)$ is real-analytic from the left of 0 with $B^{\prime}(-0)<0$ and it has a square-root singularity from the right of 0 .

Proof of Lemma 7.1. For $z=x+i y$ with $y>0$ we have from (7.1) that

$$
b^{\prime \prime}(z)=-4 \pi^{2} \sum_{m=1}^{\infty} m^{1 / 2} \exp (2 \pi i m z)=-4 \pi^{2} \sum_{m=-\infty}^{\infty} m_{+}^{1 / 2} \exp (2 \pi i m z) .
$$

Define for $z \in \mathbb{C}$ with $\operatorname{Im} z>0$,

$$
\beta(z)=\int_{-\infty}^{\infty} m_{+}^{1 / 2} \exp (2 \pi i m z) d m=\int_{0}^{\infty} m^{1 / 2} \exp (2 \pi i m z) d m=\frac{i-1}{8 \pi} z^{-3 / 2}
$$

Then by the Poisson summation formula

$$
\sum_{m=1}^{\infty} m^{1 / 2} \exp (2 \pi i m z)=\sum_{m=-\infty}^{\infty} \beta(m+z), \quad \operatorname{Im} z>0
$$

hence

$$
b^{\prime \prime}(z)=\frac{1-i}{2} \pi \sum_{m=-\infty}^{\infty}(z+m)^{-3 / 2}, \quad \operatorname{Im} z>0 .
$$

This formula defines $b^{\prime \prime}(z)$ as an analytic function in the strip $\{z=x+i y: 0<$ $x<1\}$. Now from (7.2) we obtain

$$
B^{\prime \prime}(x)=\operatorname{Im}\left(\frac{1-i}{\sqrt{2}} b^{\prime \prime}(x)\right)=-\frac{\pi}{\sqrt{2}} \sum_{m=0}^{\infty}(m+x)^{-3 / 2}, \quad 0<x<1 .
$$

This proves that $B^{\prime \prime}(x)$ (and hence $B(x)$ ) is a real analytic function on $(0,1)$ and $B^{\prime \prime}(x)<0$ so that $B(x)$ is concave.

Equation (7.3) follows directly from (7.1), and this implies (7.4) and (7.5). Finally, (7.6) follows from (7.11). Lemma 7.1 is proved.

Introduce the constants $B_{ \pm}$and $D_{ \pm}$as follows:

$$
\begin{aligned}
& B_{+}=B\left(x_{\max }\right), \quad B_{-}=-B(0) ; \\
& D_{+}=-B^{\prime \prime}\left(x_{\max }\right), \quad D_{-}=-B^{\prime}(-0),
\end{aligned}
$$

and consider the function

$$
\varphi(\lambda)=\int_{0}^{1} \exp (i \lambda B(x)) d x
$$

Lemma 7.2. $\varphi(\lambda)$ is an entire function for which

$$
\begin{aligned}
& \varphi(0)=1, \quad \varphi^{\prime}(0)=0 \\
& \varphi(\lambda)>0, \operatorname{Re} \varphi^{\prime}(\lambda)=0, \varphi^{\prime \prime}(\lambda)<0 \quad \text { if } \operatorname{Re} \lambda=0 \\
& |\varphi(\mu+i v)| \leqq \varphi(i v) \quad \forall(\mu+i v) \in \mathbb{C} .
\end{aligned}
$$


Proof. Equation (7.13) implies that $\varphi(\lambda)$ is an entire function and

$$
\begin{aligned}
\varphi^{\prime}(\lambda) & =i \int_{0}^{1} B(x) \exp (i \lambda B(x)) d x \\
\varphi^{\prime \prime}(\lambda) & =-\int_{0}^{1} B^{2}(x) \exp (i \lambda B(x)) d x,
\end{aligned}
$$

so Lemma 7.2 follows.

Corollary 7.3. The function $\varphi($ it $)$ is a strictly convex positive function of $t \in \mathbb{R}$ which achieves it minimum at $t=0$.

Lemma 7.4. The following asymptotics holds as $|\lambda| \rightarrow \infty$ : if $\lambda=\mu+i v$ with $v \geqq 0$, then

$$
\varphi(\lambda)=i\left(D_{-} \lambda\right)^{-1} e^{-i \lambda B_{-}}\left(1+O\left(|\lambda|^{-1}+|\lambda|^{1 / 2} e^{-c v}\right)\right),
$$

where $c>0$ is independent of $\lambda$; if $v \leqq 0$ then

$$
\varphi(\lambda)=(2 \pi)^{1 / 2}\left(i \lambda D_{+}\right)^{-1 / 2} e^{i \lambda B_{+}}\left(1+O\left(|\lambda|^{-1}\right)\right) .
$$

Corollary 7.5. As $|\lambda|=|\mu+i v| \rightarrow \infty$,

$$
\left|\frac{\varphi(\mu+i v)}{\varphi(i v)}\right|=O\left(\left|\frac{\mu+i v}{\mu}\right|^{-1 / 2}\right) \text {. }
$$

Proof of Lemma 7.4. Let $\chi(x)$ be a $C^{\infty}$ function which is equal to 1 in $\varepsilon$-neighborhood of the point $x_{\max }$ and which is equal to 0 outside of $2 \varepsilon$-neighborhood of $x_{\max }$. Then $\varphi(\lambda)=\varphi_{1}(\lambda)+\varphi_{2}(\lambda)+\varphi_{3}(\lambda)$, where

$$
\begin{aligned}
\varphi_{1}(\lambda) & =\int_{0}^{1} \chi(x) e^{i \lambda B(x)} d x, \\
\varphi_{2}(\lambda) & =\int_{0}^{x_{\max }}[1-\chi(x)] e^{i \lambda B(x)} d x, \\
\varphi_{3}(\lambda) & =\int_{x_{\max }}^{1}[1-\chi(x)] e^{i \lambda B(x)} d x .
\end{aligned}
$$

Let us first evaluate $\varphi_{1}(\lambda)$.

Assume $v \geqq 0$. Let $y=y(x)$ be a smooth increasing function near $x_{\max }$ such that

$$
y^{2}=B\left(x_{\max }\right)-B(x)=B_{+}-B(x) .
$$

Then

$$
\varphi_{1}(\lambda)=e^{i \lambda B_{+}} \int_{-\infty}^{\infty} \chi(y) e^{-i \lambda y^{2}} x^{\prime}(y) d y
$$

We can write

$$
x^{\prime}(y)=t+y s(y), \quad t=\left(\left|B^{\prime \prime}\left(x_{\max }\right)\right| / 2\right)^{-1 / 2}=\left(D_{+} / 2\right)^{-1 / 2}
$$

with smooth $s(y)$, hence

$$
\varphi_{1}(\lambda)=e^{i \lambda B_{+}}\left[t \varphi_{4}(\lambda)+\varphi_{5}(\lambda)\right]
$$


where

$$
\begin{aligned}
& \varphi_{4}(\lambda)=\int_{-\infty}^{\infty} \chi(y) e^{-i \lambda y^{2}} d y \\
& \varphi_{5}(\lambda)=\int_{-\infty}^{\infty} \chi(y) e^{-i \lambda y^{2}} y s(y) d y .
\end{aligned}
$$

Now we can write $\varphi_{4}(\lambda)$ as follows: $\varphi_{4}(\lambda)=\varphi_{6}(\lambda)+\varphi_{7}(\lambda)$ with

$$
\begin{aligned}
& \varphi_{6}(\lambda)=\int_{-\infty}^{\infty} \chi(y) e^{-i \mu y^{2}} d y, \\
& \varphi_{7}(\lambda)=\int_{-\infty}^{\infty} \chi(y) e^{-i \mu y^{2}}\left(e^{v y^{2}}-1\right) d y,
\end{aligned}
$$

and

$$
\begin{aligned}
& \varphi_{6}(\lambda)=O\left((1+|\mu|)^{-1 / 2}\right), \\
& \varphi_{7}(\lambda)=O\left((1+|\mu|)^{-1} e^{\varepsilon_{0} v}\right),
\end{aligned}
$$

where

$$
\varepsilon_{0}=2 \max _{\left|x-x_{\max }\right| \leqq 2 \varepsilon}|y(x)|^{2} .
$$

Indeed, (7.24) is obvious when $|\mu| \leqq 1$ and when $|\mu|>1$,

$$
\int_{-\infty}^{\infty} \chi(y) e^{-i \mu y^{2}} d y=\int_{-\infty}^{\infty} e^{-i \mu y^{2}} d y+\int_{-\infty}^{\infty}[1-\chi(y)] e^{-i \mu y^{2}} d y=c_{0}|\mu|^{-1 / 2}+O\left(|\mu|^{-1}\right)
$$

which implies (7.24). Also (7.25) is obvious when $|\mu| \leqq 1$, and when $|\mu|>1$ we integrate by parts in (7.23) which gives

$$
\varphi_{7}(\lambda)=\int_{-\infty}^{\infty} e^{-i \mu y^{2}}\left(\chi(y) \frac{e^{v y^{2}}-1}{2 i \mu y}\right)^{\prime} d y=O\left(|\mu|^{-1} e^{\varepsilon_{0} v}\right)
$$

Thus (7.24) and (7.25) are proved. Since also

$$
\varphi_{5}(\lambda)=(2 i \lambda)^{-1} \int_{-\infty}^{\infty} e^{-i \lambda y^{2}}[\chi(y) s(y)]^{\prime} d y=O\left(|\lambda|^{-1} e^{\varepsilon_{0} v}\right)
$$

we obtain from $(6.22)-(6.25)$ that

$$
\varphi_{1}(\lambda)=O\left((1+|\mu|)^{-1 / 2} e^{\left(-B_{+}+\varepsilon_{0}\right) v}\right) .
$$

We may assume $\varepsilon_{0}$ is small, say, $\varepsilon_{0}<B_{+} / 2$. Then the last relation implies

$$
\varphi_{1}(\lambda)=O\left(|\lambda|^{-1 / 2} e^{B_{-} v}\right)
$$

Let us estimate now $\varphi_{2}(\lambda)$.

By Lemma 7.1 $B(x)$ is a smooth increasing function on the interval $0<x<$ $x_{\max }-\varepsilon$ with $B^{\prime}(x)>0$ and $B^{\prime}(x)$ has a square-root singularity at $x=0$ (see (7.6)). 
Therefore $B_{0}(y)=B\left(y^{2}\right)$ is a smooth increasing function with $B_{0}^{\prime}(y)>0$ on $\left[0, y_{0}\right]$, where $y_{0}=\left(x_{\max }-\varepsilon\right)^{2}$. Making the change of variable $x=y^{2}$ in (7.21) we have

$$
\varphi_{2}(\lambda)=\int_{0}^{y_{0}}\left[1-\chi\left(y^{2}\right)\right] e^{i \lambda B_{0}(y)} 2 y d y .
$$

Since $B_{0}^{\prime}(y)>0$ we can integrate by parts twice in the last formula, integrating the exponent and differentiating the rest. The main contribution comes from the boundary term at $y=0$ and this gives

$$
\varphi_{2}(\lambda)=O\left(|\lambda|^{-2} e^{B_{-} v}\right) .
$$

Similarly, the function $B(x)$ is a smooth decreasing function on $\left[x_{\max }+\varepsilon, 1\right]$ with $B^{\prime}(x)<0$. So we can differentiate by parts in the third formula in (7.21), integrating the exponent and differentiating the rest. The main contribution comes from the boundary term at $x=1$ and this gives

$$
\varphi_{3}(\lambda)=i\left(D_{-} \lambda\right)^{-1} e^{-i \lambda B_{-}}\left(1+O\left(|\lambda|^{-1}\right)\right) .
$$

From (7.26)-(7.28), (7.19) follows. Equation (7.20) is proved similarly. Lemma 7.4 is proved.

\section{References}

1. Berry, M.V., Mount, K.E.: Semiclassical approximations in wave mechanics. Rep. Progr. Phys. 35, 315-397 (1972)

2. Berry, M.V., Tabor, M.: Closed orbits and the regular bound spectrum. Proc. R. Soc. Lond. A349, 101-123 (1976)

3. Bleher, P.M.: Quasiclassical expansion and the problem of quantum chaos. Lect. Notes in Math 1469, 60-89 (1991)

4. Bleher, P.M.: On the distribution of the number of lattice points inside a family of convex ovals. Duke Math. J. 67, 461-481 (1993)

5. Bleher, P.M.: Distribution of the error in the Weyl asymptotics for the Laplace operator on a two-dimensional torus and related lattice problems. Duke Math. J. 70, 655-682 (1993)

6. Bleher, P.M.: Distribution of energy levels of a quantum free particle on a surface of revolution. Duke Math. J. 74, 1-49 (1994)

6a. Bleher, P.M.: Semiclassical quantization rules near separatrices. Commun. Math. Phys. 165, 621-640 (1994)

7. Bleher, P.M., Cheng, Zh., Dyson, F.J., Lebowitz, J.L.: Distribution of the error term for the number of lattice points inside a shifted circle. Commun. Math. Phys. 154, 433-469 (1993)

8. Colin de Verdiére, Y.: Spectre du laplacien et longueurs des geodesiques periodiques. Comp. Math. 27, 159-184 (1973)

9. Duistermaat, J.J., Guillemin, V.: Spectrum of elliptic operators and periodic bicharacteristics. Invent. Math. 29, 39-79 (1975)

10. Guillemin, V.: Lectures on spectral theory of elliptic operators. Duke Math. J. 44, 485-517 (1977)

11. Gutzwiller, G.: Chaos in classical and quantum mechanics. New York: Springer-Verlag, 1990

12. Heath-Brown, D.R.: The distribution and the moments of the error term in the Dirichlet divisor problem. Acta Arithm. 60, 389-415 (1992)

13. Hörmander, L.: Fourier integral operators I. Acta Math. 127, 79-183 (1971)

14. Hörmander, L.: The spectral function of an elliptic operator. Acta Math. 121, 193-218 (1968)

15. Kosygin, D.V., Minasov, A.A., Sinai, Ya.G.: Statistical properties of the Laplace-Beltrami operator on Liouville surfaces. Uspekhi Mat. Nauk 48, no. 4, 3-130 (1993)

16. Lax, P., Phillips, R.: Scattering theory for automorphic functions. Princeton, NJ: Princeton University Press, 1976 
17. Levitan, B.M., Zhikov, V.V.: Almost periodic functions and differential equations. Cambridge: Cambridge Univ. Press, 1968

18. McKean, H.B.: Selberg's trace formula as applied to a compact Riemann surface. Comm. Pure Appl. Math. 25, 225-246 (1972)

19. Rubinstein, M., Sarnak, P.: Chebyshev bias (to appear)

20. Sarnak, P.: Arithmetic quantum chaos. Schur Lectures, Tel Aviv, 1992

21. Sinai, Ya.G.: Mathematical problems in the theory of quantum chaos. Lect. Notes in Math. 1469, 41-59 (1991)

22. Nguen Tien Zung, Polyakova L.S., Selivanova, E.N.: Topological classification of integrable geodesic flows with additional quadratic or linear in momenta integral on two-dimensional oriented riemannian manifolds. Funct. Analysis and Appl. 27, 42-56 (1993)

Communicated by A. Jaffe 
